\title{
Computational Methods for a Curved Beam With Piezoceramic PATCHES ${ }^{1}$
}

\author{
H.T. Banks and Y. Zhang \\ Center for Research in Scientific Computation \\ Department of Mathematics \\ North Carolina State University \\ Raleigh, NC 27695-8205
}

April 25, 1997

\begin{abstract}
We develop a computational scheme to approximate the solutions of a mathematical model that describes the vibrations of a curved beam structure. The physical model also includes pairs of piezoceramic patches that can be viewed either as passive devices, changing only the structural properties of the beam, or as active devices used to control vibrations of the beam. As part of our numerical experiments, we compare schemes with a method that employs hybrid B-spline basis elements and a method that employs just one family of B-spline basis elements. We calculate the first four fundamental frequencies and mode shapes of the structure, and we simulate patch-activated vibrations. Finally, we devote a section to investigations of locking phenomena which have been reported for some shell problems.
\end{abstract}

\footnotetext{
${ }^{1}$ This research was supported in part by the U.S. Air Force Office of Scientific Research under grants AFOSR F49620-95-1-0236 and AFOSR F49620-93-1-0280 and in part by the U.S. Department of Education through a GAANN Fellowship to Y.Z. under grant P200A40730.
} 


\section{Introduction}

In recent years a rapid rise in interest in piezoceramic activated and sensed structures can be found in the context of smart material structures ([ACCHW, ATW, CA, CFW, CL, DFR, DIG, FGS, FH, GT, HF, RWTW]). While much of the literature reports on efforts with flat structures such as beams and plates, there is acknowledged practical interest in structures with curvature (curved cylindrical sections, cylindrical shells, etc.) since these arise in many applications involving automobile, aircraft and submarine surfaces ([JR, TG]). There are a number of important differences between piezo actuation of flat surfaces and similar-but-curved surfaces. Among them is the simple fact that it is often reasonable to assume that it is possible to excite a flat structure (with pairs of patches actuated either "in phase" or "out-ofphase") so as to decouple transverse and longitudinal (in plane) vibrations, thereby greatly simplifying investigations of active control. However for curved structures, the coupling between transverse (radial) and longitudinal (tangential) motions is inherent and one, in general, cannot excite the structures so as to decouple them.

In [BMZ, BSW], the authors develop detailed models for curved structures with surface bonded piezoceramic patch actuators. The models include both passive and active contributions of the patches to the overall structural model and parameters such as stiffness, damping, etc. A convergence theory for Galerkin type finite element approximation schemes for curved beams with piezo actuators is presented in [BMZ]. Here we report on computational investigations of two such schemes to indicate some of their possible advantages and shortcomings. We use these to illustrate numerically several features (system theoretic aspects involving coupled motions, changes in fundamental frequencies and mode shapes due to passive patch contributions, locking type behavior, etc.) of curved structures with piezo actuators. For example, we shall find below that we must consider coupled "system" frequencies and mode shapes which are not closely related to the modes of an associated flat surface. Moreover, even small patches "load" the structure with respect to stiffness (and less so, with respect to mass density) and hence the frequencies and mode shapes are substantially different from those of a similar curved structure without patches.

Since locking type phenomena are known to occur in numerical calculations for curved and shell-like structures, we present a brief section with numerical examples to illustrate this aspect of the computational methods we use. However, this paper is not about "locking" in finite element methods and we present the brief discussions on the subject here only because it is such a common occurrence in methods for curved structures such as arches and shells. We do present enough to illustrate possible difficulties and possible remedies (here we illustrate a type of "mixed-method" fix-up to locking which allows one to satisfy certain limiting constraints in some weaker sense). For a more complete study of locking in curved arches, the readers should consult $[\mathrm{Z}]$ and the numerous references cited there. 
After a brief summary of the model including passive and active patch contributions in Section 2, we describe in Section 3 a family of spline based approximation schemes. Semidiscretization (discretization in the spatial variable) with these schemes yields high order approximating ordinary differential equation systems. These systems are used in Section 4 in numerical simulations to compare computational accuracy with expected rates of convergences suggested by the general theory of spline approximations. We also compute frequencies and mode shapes for comparison with those of corresponding systems with the passive patch contributions ignored.

Finally, we complete our contribution here with a discussion of locking phenomena in Section 5.

\section{Model Development}

\subsection{Physical Model and Assumptions}

The physical model under consideration is assumed to have the shape of an arc cut from the cross-section of a cylindrical shell. The curved beam structure therefore has finite curvature, $R$, in the direction of its length, $l_{b}=R\left(\theta_{2}-\theta_{1}\right)$. The smart material patches, piezoceramic patches, that are bonded to this parent beam have the same width and the same curvature as the beam, but with length $l_{p}=R\left(\theta_{1}^{p}-\theta_{2}^{p}\right)$. We require $\left[\theta_{1}^{p}, \theta_{2}^{p}\right] \subset\left[\theta_{1}, \theta_{2}\right]$. As is common practice in the smart material literature, we include pairs of identical patches to produce a symmetric geometry about the midsurface. A view of the patched beam from the side is given in Figure 2.1, where $h$ denotes the thickness of the beam and each patch is of thickness $T$.

The transverse vibrations of the patched beam are usually the primary consideration. However, due to the beam curvature there are two displacements involved: $v(t, \theta)$ in the tangential $(\hat{\theta})$ direction and $w(t, \theta)$ in the radial $(\hat{n})$ direction. Any motion in the direction $(\hat{x})$ of the width of the beam, is assumed to be negligible. As a consequence, the Poisson ratios are also neglected. Moreover, we make the following assumptions for our model:

- Each material layer is bonded perfectly to others in contact, i.e., no slip and no disbonds are allowed.

- Each material layer deforms uniformly with respect to thickness; that is, displacements are only functions of $t$ and $\theta$.

- The thickness of the patched beam is very small in comparison with the other dimensions such as length of the beam.

- The deformations are sufficiently small so as to allow higher order motions to be neglected, i.e., first order models are used. 
- Transverse normal stresses (i.e., the stresses in the direction, $\hat{n}$, normal to the length) are taken to be negligible.

- A cross section which is originally normal to the beam reference surface will remain normal to the deformed reference surface and will remain unstrained (i.e., no shear, no rotation).

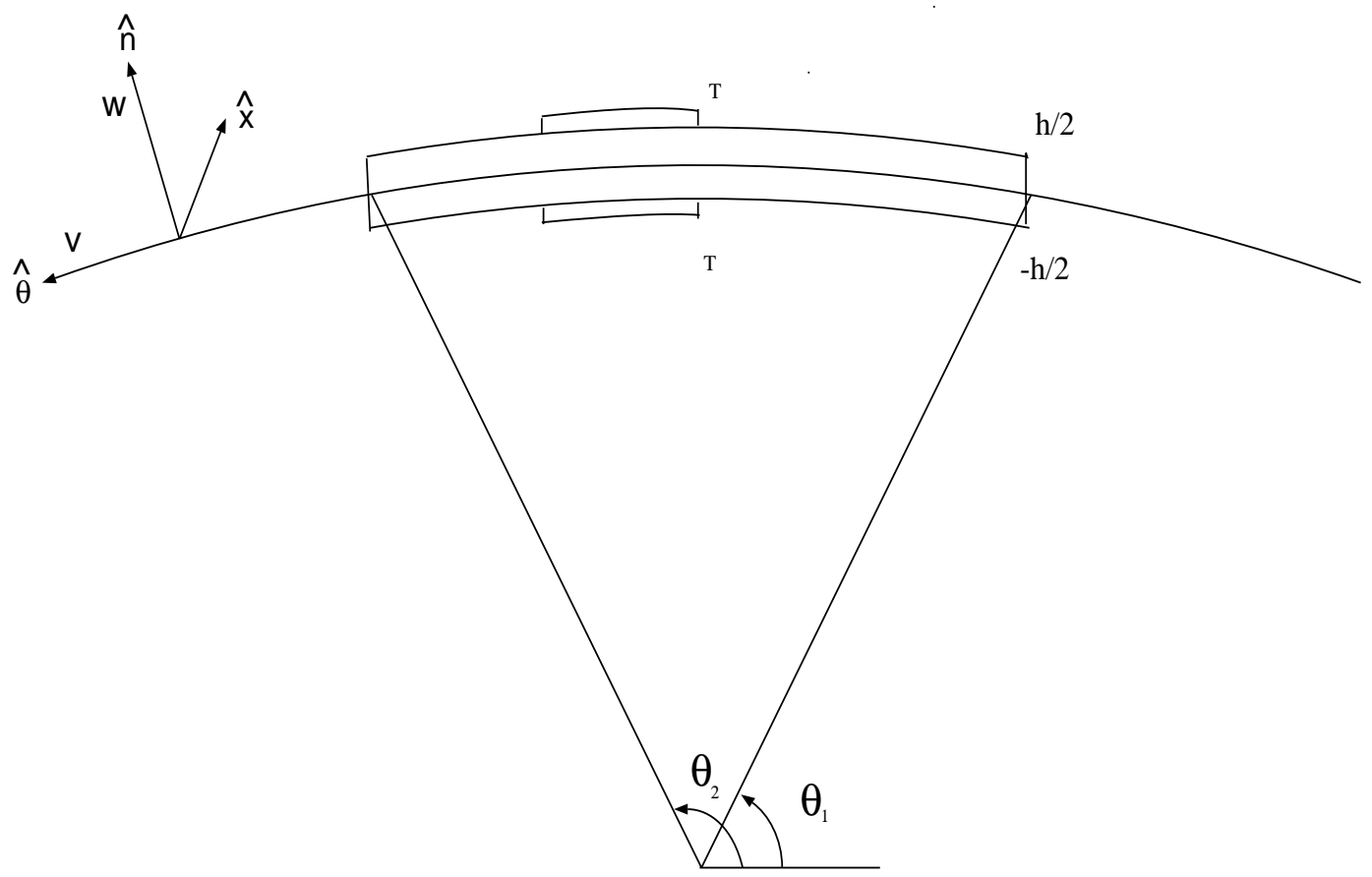

Figure 2.1 Composite Curved Beam

These assumptions can also be found in [BMZ], where well-posedness and theoretical convergence analysis for general approximation schemes for a similar structure are presented.

\subsection{Mathematical Model}

The formulation of our model for the curved beam is based on the Donnell-Mushtari theory for shell models, and the general derivations are given in [BSW]. The tangential and the radial displacements, $v$ and $w$, of a beam of width $b$ and length $l_{b}$ are given by

$$
\begin{aligned}
R \bar{\rho} \frac{\partial^{2} v}{\partial t^{2}}-b \frac{\partial N_{\theta}}{\partial \theta} & =b R \hat{q}_{\theta} \\
R \bar{\rho} \frac{\partial^{2} w}{\partial t^{2}}-\frac{b}{R} \frac{\partial^{2} M_{\theta}}{\partial \theta^{2}}+b N_{\theta} & =b R \hat{q}_{n}+b \frac{\partial \hat{m}_{x}}{\partial \theta}
\end{aligned},
$$

for $t>0, \theta_{1}<\theta<\theta_{2}$, where $N_{\theta}$ is the total internal force in the $\hat{\theta}$ direction, $M_{\theta}$ is the total internal moment resultant about the $\hat{x}$ direction, $\hat{q}_{\theta}$ is the total applied line 
force in the $\hat{\theta}$ direction, $\hat{q}_{n}$ is the total applied line force in the $\hat{n}$ direction, and $\hat{m}_{x}$ is the total applied line moment in the $\hat{x}$ direction. In these equations, $\bar{\rho}$ is the linear mass density which varies with $\theta$ due to the presence of the patches which do not completely cover the beam. If we take $\rho^{e}, \rho^{p}$ to be the volume mass densities of the elastic and piezoceramic material, respectively, then we have

$$
\bar{\rho}=\rho^{e} h b+\rho^{p} 2 T b \chi_{p},
$$

where $h$ and $T$ are the thicknesses of the elastic beam and each piezo patch, respectively, and $\chi_{p}=\chi_{p}(\theta)$ is the characteristic function for the patch (i.e., it is 1 on regions where the patch is present and 0 elsewhere).

Furthermore, we assume clamped-ends boundary conditions so that

$$
\begin{aligned}
v\left(t, \theta_{1}\right) & =v\left(t, \theta_{2}\right)=0 \\
w\left(t, \theta_{1}\right) & =w\left(t, \theta_{2}\right)=0 \\
\frac{\partial w}{\partial \theta}\left(t, \theta_{1}\right) & =\frac{\partial w}{\partial \theta}\left(t, \theta_{2}\right)=0
\end{aligned} .
$$

\subsubsection{Equations of motion}

The total internal force and total internal moment resultants required in (2.1) are deduced in $[\mathrm{BMZ}]$ to be:

$$
\begin{aligned}
N_{\theta}= & \frac{E^{e} h}{R}\left(\frac{\partial v}{\partial \theta}+w\right)+\frac{C_{D}^{e} h}{R}\left(\frac{\partial^{2} v}{\partial t \partial \theta}+\frac{\partial w}{\partial t}\right) \\
& +\frac{E^{p} 2 T}{R}\left(\frac{\partial v}{\partial \theta}+w\right) \chi_{p}(\theta)+\frac{C_{D}^{p} 2 T}{R}\left(\frac{\partial^{2} v}{\partial t \partial \theta}+\frac{\partial w}{\partial t}\right) \chi_{p}(\theta) \\
M_{\theta}= & -\frac{E^{e} h^{3}}{12 R^{2}} \frac{\partial^{2} w}{\partial \theta^{2}}-\frac{C_{D}^{\epsilon} h^{3}}{12 R^{2}} \frac{\partial^{3} w}{\partial t \partial \theta^{2}} \\
& -\frac{E^{p} 2 \mu_{1}}{3 R^{2}} \frac{\partial^{2} w}{\partial \theta^{2}} \chi_{p}(\theta)-\frac{C_{D}^{p} 2 \mu_{1}}{3 R^{2}} \frac{\partial^{3} w}{\partial t \partial \theta^{2}} \chi_{p}(\theta),
\end{aligned}
$$

where $\mu_{1}=\left(\frac{h}{2}+T\right)^{3}-\left(\frac{h}{2}\right)^{3}$. Here $E^{e}, E^{p}, C_{D}^{e}$ and $C_{D}^{p}$ are the Young's moduli of the beam and the piezoceramic material, the Kelvin-Voigt damping coefficient of the beam and the piezoceramic material, respectively. To simplify our notation, we adopt the notational conventions that $\partial=\frac{\partial}{\partial \theta}$ and an overdot represents $\frac{\partial}{\partial t}$. We also define the following functions:

$$
\begin{aligned}
& \xi_{1}(\theta)=-\frac{b}{R^{2}}\left(E^{e} h+2 E^{p} T \chi_{p}(\theta)\right) \\
& \xi_{2}(\theta)=\frac{b}{R^{4}}\left(\frac{E^{e} h^{3}}{12}+\frac{2 E^{p} \mu_{1}}{3} \chi_{p}(\theta)\right) \\
& \xi_{3}(\theta)=-\frac{b}{R^{2}}\left(C_{D}^{e} h+2 C_{D}^{p} T \chi_{p}(\theta)\right) \\
& \xi_{4}(\theta)=\frac{b}{R^{4}}\left(\frac{C_{D}^{e} h^{3}}{12}+\frac{2 C_{D}^{p} \mu_{1}}{3} \chi_{p}(\theta)\right) .
\end{aligned}
$$


Using the forces, moments and variables defined above, we may rewrite system (2.1) as

$$
\begin{gathered}
\bar{\rho} \ddot{v}+\partial\left(\xi_{1}(\partial v+w)\right)+\partial\left(\xi_{3}(\partial \dot{v}+\dot{w})\right)=b \hat{q}_{\theta} \\
\bar{\rho} \ddot{w}+\partial^{2}\left(\xi_{2}\left(\partial^{2} w\right)\right)-\xi_{1}(\partial v+w)+\partial^{2}\left(\xi_{4}\left(\partial^{2} \dot{w}\right)\right)-\xi_{3}(\partial \dot{v}+\dot{w})=b \hat{q}_{n}+\frac{b}{R} \partial \hat{m}_{x}
\end{gathered}
$$

where, when appropriate, derivatives must be interpreted in the distributional sense. For more complete discussions of the distributional form of these equations, see [BMZ] and [BSW].

\subsubsection{External forces and moments}

For our choices of the external forces and moments, we use a form such as those produced by PZT piezoceramic patches. To aid in quantitative description of the PZT forces, we define the indicator-function

$$
\hat{S}_{1,2}(\theta)=\left\{\begin{array}{ll}
1 & \theta>\left(\theta_{1}^{p}+\theta_{2}^{p}\right) / 2 \\
0 & \theta=\left(\theta_{1}^{p}+\theta_{2}^{p}\right) / 2 \\
-1 & \theta<\left(\theta_{1}^{p}+\theta_{2}^{p}\right) / 2
\end{array} .\right.
$$

Again, we are following the notations and formulations in [BSW] and [BSW1]. For our model, if we assume that the two piezo patches have the same Young's modulus, $E^{p}$ and the same dimensions, and if we define $K_{B}=E^{p} d_{31}(h+T)$, we obtain the following model that describes vibrations of a patch-activated curved beam:

$$
\begin{gathered}
\bar{\rho} \ddot{v}+\partial\left(\xi_{1}(\partial v+w)\right)+\partial\left(\xi_{3}(\partial \dot{v}+\dot{w})\right)=-\frac{b}{R} \hat{S}_{1,2}(\theta) \partial \hat{N}_{\theta} \\
\bar{\rho} \ddot{w}+\partial^{2}\left(\xi_{2}\left(\partial^{2} w\right)\right)-\xi_{1}(\partial v+w)+\partial^{2}\left(\xi_{4}\left(\partial^{2} \dot{w}\right)\right)-\xi_{3}(\partial \dot{v}+\dot{w})=-\frac{b}{R^{2}} \partial^{2} \hat{M}_{\theta}
\end{gathered}
$$

where

$$
\begin{aligned}
b \hat{N}_{\theta} & =-\frac{b K_{B}}{h+T}\left(V_{1}+V_{2}\right) \chi_{p}(\theta) \hat{S}_{1,2}(\theta) \\
b \hat{M}_{\theta} & =-\frac{b}{2} K_{B}\left(V_{1}-V_{2}\right) \chi_{p}(\theta)
\end{aligned}
$$

The latter are the forces and moments due to the piezoceramic patches which deform (expand or contract) in response to the applied voltages $V_{1}(t)$ and $V_{2}(t)$. These forces and moments involve $\hat{q}_{\theta}$ and $\hat{m}_{x}$ on the right sides of the equations in (2.3). The term, $\hat{q}_{n}$, can be an axisymmetric load on the beam, external air damping, etc.; however, when the patches are the only active agent, $\hat{q}_{n}=0$. General well-posedness theorems for the resulting system (with unbounded inputs and coefficients) in weak or variational form follow easily from results presented elsewhere (see [BIW] and [BSW]) as discussed in the next section. 


\section{Spline Approximation Schemes}

To provide numerical approximations to the solutions of the infinite dimensional model, system (2.3), or system (2.4), we apply a Galerkin approximation scheme. That is, we first give the weak formulations of the above models, then define finite approximation spaces that contain appropriate trial basis functions and use these basis elements to formulate a finite dimensional model to approximate the original system. The corresponding necessary convergence results are presented in [BMZ].

Before presenting the weak formulations, we perform a change of a variable so that the independent variable, $\theta$, is defined on $[0,1]$ instead of $\left[\theta_{1}, \theta_{2}\right]$. The transformation is given by: $\tilde{\theta}=\frac{\theta-\theta_{1}}{\theta_{2}-\theta_{1}}$. We make this change of variable in (2.3) and (2.4), and again to simplify notation, we use the symbol $\theta$ to denote the new variable as well. If we define $\ell=\theta_{2}-\theta_{1}$, the system $(2.3)$ then becomes

$$
\begin{aligned}
& \bar{\rho} \ddot{v}+\frac{1}{\ell} \partial\left(\xi_{1}\left(\frac{1}{\ell} \partial v+w\right)\right)+\frac{1}{\ell} \partial\left(\xi_{3}\left(\frac{1}{\ell} \partial \dot{v}+\dot{w}\right)\right)=b \hat{q}_{\theta} \\
& \bar{\rho} \ddot{w}+\frac{1}{\ell^{2}} \partial^{2}\left(\xi_{2}\left(\frac{1}{\ell^{2}} \partial^{2} w\right)\right)-\xi_{1}\left(\frac{1}{\ell} \partial v+w\right) \\
&+\frac{1}{\ell^{2}} \partial^{2}\left(\xi_{4}\left(\frac{1}{\ell^{2}} \partial^{2} \dot{w}\right)\right)-\xi_{3}\left(\frac{1}{\ell} \partial \dot{v}+\dot{w}\right)=b \hat{q}_{n}+\frac{b}{\ell R} \partial \hat{m}_{x}
\end{aligned}
$$

with scaled arbitrary external forces and moments. We shall not write out the similar scaled analogue of (2.4).

To illustrate the changes on the right sides of the latter system, we present the weak formulations of the model with active patches:

$$
\begin{aligned}
\int_{0}^{1}\left(\bar{\rho} \ddot{v} \phi-\frac{\xi_{1}}{\ell}\left(\frac{1}{\ell} \partial v+w\right) \phi^{\prime}-\frac{\xi_{3}}{\ell}\left(\frac{1}{\ell} \partial \dot{v}+\dot{w}\right) \phi^{\prime}\right) d \theta & =\int_{0}^{1} \frac{b}{\ell R} \hat{N}_{\theta} \phi^{\prime} d \theta \\
\int_{0}^{1}\left(\bar{\rho} \ddot{w} \psi+\frac{\xi_{2}}{\ell^{2}}\left(\frac{1}{\ell^{2}} \partial^{2} w\right) \psi^{\prime \prime}-\xi_{1}\left(\frac{1}{\ell} \partial v+w\right) \psi\right. & \\
\left.+\frac{\xi_{4}}{\ell^{2}}\left(\frac{1}{\ell^{2}} \partial^{2} \dot{w}\right) \psi^{\prime \prime}-\xi_{3}\left(\frac{1}{\ell} \partial \dot{v}+\dot{w}\right) \psi\right) d \theta & =-\int_{0}^{1} \frac{b}{\ell^{2} R^{2}} \hat{M}_{\theta} \psi^{\prime \prime} d \theta .
\end{aligned}
$$

The pair $(\phi, \psi) \in \mathcal{V}=H_{0}^{1}(0,1) \times H_{0}^{2}(0,1)$ are the test functions used to define the above system. As usual, they satisfy the boundary conditions that are imposed on $v(\cdot, \theta)$ and $w(\cdot, \theta)$ in $(2.2)$. (Note that the first equation in the above system is not obtained by simply performing direct integrations by parts on that of (2.4); see [BSW] for details). The space $\mathcal{V}$ is a Hilbert space used to form a Gelfand triple with the pivot space $\mathcal{H}=L_{2}(0,1) \times L_{2}(0,1)$ (see [BMZ]), and is the basis of well-posedness results for general systems in [BMZ] that include (3.1) or (3.2) as special cases. More precisely, existence, uniqueness and continuous dependence of solutions to (3.1) can be obtained via semigroup theory and are given in [BMZ]. These conclusions for the equivalent weak form (3.2) also follow immediately from results in [BIW] and Chapter 4 of $[\mathrm{BSW}]$. We note (see [BIW] or Remark 4.2, p. 103 of [BSW]) that solutions are actually in $C(0, T ; \mathcal{V})$. 
Next we assume the unknowns $v(t, \theta)$ and $w(t, \theta)$ can be approximated in terms of trial functions, $\eta_{i}^{N_{v}}(\theta), i=1 \cdots N_{v}$, and $\zeta_{k}^{N_{w}}(\theta), k=1 \cdots N_{w}$, which are basis elements of the approximation spaces of dimensions $N=\left(N_{v}, N_{w}\right)$, and generalized Fourier coefficients $\bar{v}_{i}^{N_{v}}(t)$ and $\bar{w}_{k}^{N_{w}}(t)$ in the form

$$
\begin{aligned}
v^{N_{u}}(t, \theta) & =\sum_{i=1}^{N_{u}} \bar{v}_{i}^{N_{u}}(t) \eta_{i}^{N_{u}}(\theta) \\
w^{N_{w}}(t, \theta) & =\sum_{k=1}^{N_{w}} \bar{w}_{k}^{N_{w}}(t) \zeta_{k}^{N_{w}}(\theta) .
\end{aligned}
$$

The superscripts, $N_{v}$ and $N_{w}$, will be discarded whenever no confusion may result. We also assume each pair $\left(\eta_{i}, \zeta_{k}\right) \in \mathcal{V}$, as do the pair of test functions.

We substitute (the right sides of (3.1) are used to represent general external forcing functions) the finite series expressions for $v^{N_{v}}$ and $w^{N_{w}}$ into system (3.2) and use $\left(\phi_{i}, \psi_{k}\right)=\left(\eta_{i}^{N_{v}}, \zeta_{k}^{N_{w}}\right), 1 \leq i \leq N_{v}, 1 \leq k \leq N_{w}$ to denote both pairs of trial functions and test functions which are chosen to be identical. We obtain

$$
\begin{aligned}
& \int_{0}^{1} b \hat{q}_{\theta}(t, \theta) \phi_{j}(\theta) d \theta=\int_{0}^{1} \bar{\rho} \sum_{i=1}^{N_{v}} \ddot{\bar{v}}_{i}(t) \phi_{i}(\theta) \phi_{j}(\theta) d \theta \\
& -\int_{0}^{1} \frac{\xi_{1}}{\ell^{2}} \sum_{i=1}^{N_{v}} \bar{v}_{i}(t) \phi_{i}^{\prime}(\theta) \phi_{j}^{\prime}(\theta) d \theta-\int_{0}^{1} \frac{\xi_{3}}{\ell^{2}} \sum_{i=1}^{N_{v}} \dot{\bar{v}}_{i}(t) \phi_{i}^{\prime}(\theta) \phi_{j}^{\prime}(\theta) d \theta \\
& -\int_{0}^{1} \frac{\xi_{1}}{\ell} \sum_{k=1}^{N_{w}} \bar{w}_{k}(t) \psi_{k}(\theta) \phi_{j}^{\prime}(\theta) d \theta-\int_{0}^{1} \frac{\xi_{3}}{\ell} \sum_{k=1}^{N_{w}} \dot{\bar{w}}_{k}(t) \psi_{k}(\theta) \phi_{j}^{\prime}(\theta) d \theta \\
& \int_{0}^{1} b\left(\hat{q}_{n}(t, \theta)+\frac{\partial \hat{m}_{x}(t, \theta)}{\ell R}\right) \psi_{m}(\theta) d \theta=\int_{0}^{1} \bar{\rho} \sum_{k=1}^{N_{w}} \ddot{\bar{w}}_{k}(t) \psi_{k}(\theta) \psi_{m}(\theta) d \theta \\
& -\int_{0}^{1} \frac{\xi_{1}}{\ell} \sum_{i=1}^{N_{v}} \bar{v}_{i}(t) \phi_{i}^{\prime}(\theta) \psi_{m}(\theta) d \theta-\int_{0}^{1} \frac{\xi_{3}}{\ell} \sum_{i=1}^{N_{v}} \dot{\bar{v}}_{i}(t) \phi_{i}^{\prime}(\theta) \psi_{m}(\theta) d \theta \\
& -\int_{0}^{1} \xi_{1} \sum_{k=1}^{N_{w}} \bar{w}_{k}(t) \psi_{k}(\theta) \psi_{m}(\theta) d \theta-\int_{0}^{1} \xi_{3} \sum_{k=1}^{N_{w}} \dot{\bar{w}}_{k}(t) \psi_{k}(\theta) \psi_{m}(\theta) d \theta \\
& +\int_{0}^{1} \frac{\xi_{2}}{\ell^{4}} \sum_{k=1}^{N_{w}} \bar{w}_{k}(t) \psi_{k}^{\prime \prime}(\theta) \psi_{m}^{\prime \prime}(\theta) d \theta+\int_{0}^{1} \frac{\xi_{4}}{\ell^{4}} \sum_{k=1}^{N_{w}} \dot{\bar{w}}_{k}(t) \psi_{k}^{\prime \prime}(\theta) \psi_{m}^{\prime \prime}(\theta) d \theta
\end{aligned}
$$

Let the indices $j, m$ on $\phi_{j}$ and $\psi_{m}$ run from 1 to $N_{v}$ and $N_{w}$, respectively, and write the system in the following matrix form:

$$
M \ddot{z}+C \dot{z}+K z=F,
$$

where $z(t)=\left[\bar{v}_{1}(t) \ldots \bar{v}_{N_{v}}(t) \bar{w}_{1}(t) \ldots \bar{w}_{N_{w}}(t)\right]$. The coefficient matrices, $M, K, C$ and the vector $F$ are the so-called mass matrix, stiffness matrix, damping matrix and the 
external force vector. As an example, the mass matrix is given by:

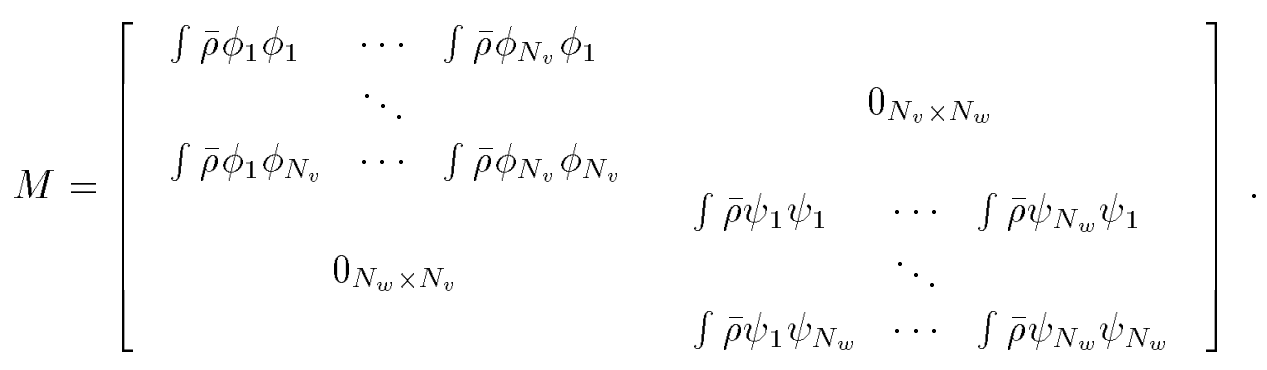

Note that the size of $M$ depends on $N=\left(N_{v}, N_{w}\right)$, as do the sizes of $K, C, F, z$. However, we suppress this index in our discussions whenever no confusion will result.

Finally, we rewrite the above matrix system as a first order differential system in time:

$$
\left[\begin{array}{c}
\dot{z} \\
\ddot{z}
\end{array}\right]=\left[\begin{array}{cc}
0 & I \\
-M^{-1} K & -M^{-1} C
\end{array}\right]\left[\begin{array}{c}
z \\
\dot{z}
\end{array}\right]+\left[\begin{array}{c}
0 \\
M^{-1} F
\end{array}\right]
$$

so that we can use stiff-ODE solvers such as the Gear method to find the solutions $v^{N_{v}}(t, \theta)$ and $w^{N_{w}}(t, \theta)$ by first evaluating $z(t)$. The next two sections contain numerical simulations of this matrix model under various physical conditions.

\section{Numerical Computation}

Within the constraints of the function spaces $\mathcal{V}$ to which the test functions $(\phi, \psi)$ belong, a number of choices are possible. For studies here, linear B-splines and cubic $\mathrm{B}$-splines are chosen to be the basis elements of the approximation spaces. The entries of the matrices in (3.6) can be readily computed in a FORTRAN subroutine with Gaussian quadrature rule. Then the IMSL subroutine, DIVPAG, which implements the Gear method with variable order, is called to solve (3.6).

\begin{tabular}{l|lll}
\hline \multirow{2}{*}{ Beam } & material: & aluminum & \\
\cline { 2 - 4 } & dimension : & width $\times$ thickness $=6.67 \mathrm{e}-2 \mathrm{ft} \times 5.21 \mathrm{e}-3 \mathrm{ft}$ & length $=1.50 \mathrm{ft}$ \\
\cline { 2 - 4 } & property & $E^{e}=1.46 \mathrm{e}+9 \mathrm{lb} / f t^{2}$ & $\rho^{e}=5.40 \mathrm{slug} / f t^{3}$ \\
\cline { 2 - 4 } & & $C_{D}^{e}=2.20 \mathrm{e}+4 \mathrm{lb} \cdot \mathrm{s} / f t^{2}$ & \\
\hline Patch & material: & PZT & $\rho^{p}=17.5 \mathrm{slug} / \mathrm{ft}^{3}$ \\
\hline & dimension : & width $\times$ thickness $=6.67 \mathrm{e}-2 \mathrm{ft} \times 8.33 \mathrm{e}-4 \mathrm{ft}$ & length $=.375 \mathrm{ft}$ \\
\cline { 2 - 4 } & property & $E^{p}=1.43 \mathrm{e}+9 \mathrm{lb} / \mathrm{ft}^{2}$ & $K_{B}=4.83 \mathrm{e}-2$ \\
\cline { 2 - 4 } & & $C_{D}^{p}=2.55 \mathrm{lb} \cdot \mathrm{s} / \mathrm{ft}^{2}$ & \\
\hline
\end{tabular}

Table 4.1: Characteristic parameters of the structure

For our computational efforts, the physical parameters (see Table 4.1) used in the mathematical model are a combination of actual lab data and handbook values that 
were obtained by colleagues in experimental work (on damaged straight beams) in the Mechanical Systems Laboratory at Virginia Tech (see [BILW], p. 32). The radius of curvature of the beam is set to be $R=1.0$ for the physical model. Before any simulations are done, systematic checking of the computer codes is necessary.

\subsection{Testing of the Codes}

To test the accuracy of the approximation and the correctness of the codes, known analytic expressions for $v(t, \theta)$ and $w(t, \theta)$ (henceforth referred to as "true solutions" and denoted by $V^{*}$ and $W^{*}$ ) are substituted in the system (3.1) to produce the analytic external forcing terms that are compatible with these solutions. Then, we can solve the approximating ODE system using these forcing terms as the known applied forces and compare the computed results to the true solutions for accuracy, numerical rates of convergence, etc. As is practice in testing numerical algorithms and codes, these true solutions were in each case chosen in a simple way so as to facilitate analytical solutions on which optimal convergence rates are usually observed.

We divide our discussions into two major subsections: the static case (with $v, w$ being constant in time) and the dynamic case of the model. For each case, the following two methods are applied: the Linear/Cubic method and the Cubic/Cubic method. The latter differs from the former in its selections of the basis elements, $\phi_{i}=\eta_{i}, i=1 \cdots N_{v}$ in (3.3). For the Linear/Cubic method, piecewise linear Bsplines, $\phi \in H_{0}^{1}(0,1)$ are chosen; while for the Cubic/Cubic method, cubic B-splines, $\phi \in H_{0}^{1} \cap H^{2}(0,1)$ are chosen. Splines which take nonzero values at $\theta=0$ or $\theta=1$ are either discarded (in the linear B-splines case) or combined linearly (in the cubic B-splines case) to satisfy the boundary conditions imposed on $v(t, \theta)$. As a result of modifying the splines, there are $N_{v}=n$ linear B-splines for the Linear/Cubic method and $N_{v}=n+2$ modified cubic B-splines for the Cubic/Cubic method, on a uniform grid with grid size $1 /(n+1)$. For the approximation of $w(t, \theta)$, both methods utilize modified cubic B-splines, $\psi_{k}=\zeta_{k} \in H_{0}^{2}(0,1), 1 \leq k \leq N_{w}=n$, defined on a uniform grid with grid size $1 /(n+1)$.

In the following experiments, we study the errors in the computed solutions by calculating the ratios of the relative errors (defined later) on an original mesh and on a mesh that has its number of mesh intervals doubled. These ratios reveal the rates of convergence for the solutions. Note that the Linear/Cubic method is a hybrid method in the sense that one family of basis elements (linear B-splines) can be expected to produce a quadratic rate of convergence and that the other family of basis elements (cubic B-splines) should produce a quartic rate of convergence under sufficient smoothness conditions on the solutions (see [BK], p. 301). 


\subsubsection{Static case}

The following true solutions were substituted into (3.1) to generate the forcing functions, which are then multiplied by basis elements and integrated over $[0,1]$ to generate the external force vector $F$, defined in (3.5). The true solutions are:

$$
\begin{aligned}
V^{*}=v(\theta) & =-0.4 \theta^{2}+0.4 \theta \\
W^{*}=w(\theta) & =16 \theta^{4}-32 \theta^{3}+16 \theta^{2} .
\end{aligned}
$$

In this case, we solve for $\hat{z}$ from $K \hat{z}=F$, where $\hat{z}=(\bar{v}, \bar{w})$ and $\bar{v}=\left(v_{1}^{N_{v}}, v_{2}^{N_{v}} \cdots v_{N_{v}}^{N_{v}}\right)$, $\bar{w}^{N_{w}}=\left(w_{1}^{N_{w}}, w_{2}^{N_{w}} \cdots w_{N_{w}}^{N_{w}}\right)$. (In the following tables, $K$-cond denotes the condition number of the stiffness matrix, $K$ ). As mentioned in the previous section, the computed solutions take the forms of

$$
\begin{aligned}
v_{c}(\theta) & =\sum_{j=1}^{N_{v}} \bar{v}_{j}^{N_{v}} \phi_{j}(\theta) \\
w_{c}(\theta) & =\sum_{j=1}^{N_{w}} \bar{w}_{k}^{N_{w}} \psi_{k}(\theta) .
\end{aligned}
$$

Each complete simulation consists of three different simulation tests:

1. set $V^{*} \neq 0, W^{*}=0$, compute $F$, solve for $\hat{z}$, and compare $v_{c}$ vs. $V^{*}$.

2. set $V^{*}=0, W^{*} \neq 0$, compute $F$, solve for $\hat{z}$, and compare $w_{c}$ vs. $W^{*}$.

3. set $V^{*} \neq 0, W^{*} \neq 0$, compute $F$, solve for $\hat{z}$, and compare $v_{c}$ vs. $V^{*}$ and $w_{c}$ vs. $W^{*}$.

When holding either $V^{*}$ or $W^{*}$ to be zero, the two equations in (2.4) are "decoupled" in $v$ and $w$ in the sense that they have minimum dependence on each other, e.g., in (3.2) a coupled product like $w \phi^{\prime}=0$ if $W^{*}=0$. Therefore, in Test 1 and Test 2, we can expect to obtain the rate of convergence for the nonzero component-quadratic rate if linear splines are employed and quartic rate if cubic splines are employedwhile holding the mesh for the approximation of the other component fixed. In Test 3 , if the Linear/Cubic method is used, we can expect to obtain quadratic rate for the convergence of the $v_{c}$ component, for which linear splines are employed. For the $w_{c}$ component, for which cubic splines are employed, we expect to obtain a convergence rate not quite quartic as the convergence in the $v_{c}$ component is only quadratic in the coupled system. If the Cubic/Cubic method is used, we expect to obtain quartic rates for both components. The relative errors are defined as: Verr $=\left\|V^{*}-v_{c}\right\| /\left\|V^{*}\right\|$ and Werr $=\left\|W^{*}-w_{c}\right\| /\left\|W^{*}\right\|$, where $\|\cdot\|$ is the sup norm over $\theta \in(0,1)$, and we test our conjectures in the following simulations. 
Simulation 1. First, we assume there are no patches bonded to the curved beam, i.e., $\chi_{p}=0$, and we apply the Linear/Cubic method for Tests $1-3$ (Table 4.2).

Test 1:

\begin{tabular}{||r|c|r|r|r||}
\hline Verr & ratio of error & $N_{v}$ & $N_{w}$ & $K$-cond \\
\hline $5.86 \mathrm{e}-02$ & & 3 & 3 & $1.22 \mathrm{e}+02$ \\
\hline $1.54 \mathrm{e}-02$ & 3.81 & 7 & 3 & $1.24 \mathrm{e}+03$ \\
\hline $3.89 \mathrm{e}-03$ & 3.96 & 15 & 3 & $8.92 \mathrm{e}+03$ \\
\hline
\end{tabular}

Test 2:

\begin{tabular}{||r|c|r|r|r||}
\hline Werr & ratio of error & $N_{v}$ & $N_{w}$ & $K$-cond \\
\hline $3.20 \mathrm{e}-03$ & & 3 & 3 & $1.20 \mathrm{e}+02$ \\
\hline $2.27 \mathrm{e}-04$ & 14.1 & 3 & 7 & $2.79 \mathrm{e}+02$ \\
\hline $1.48 \mathrm{e}-05$ & 15.3 & 3 & 15 & $3.30 \mathrm{e}+02$ \\
\hline
\end{tabular}

Test 3:

\begin{tabular}{||r|c||r|c|r|r|r||}
\hline Verr & ratio of error & Werr & ratio of error & $N_{\imath}$ & $N_{w}$ & $K$-cond \\
\hline $6.03 \mathrm{e}-02$ & & $6.69 \mathrm{e}-03$ & & 3 & 3 & $1.22 \mathrm{e}+02$ \\
\hline $1.45 \mathrm{e}-02$ & 4.16 & $2.81 \mathrm{e}-03$ & 2.38 & 7 & 7 & $1.27 \mathrm{e}+03$ \\
\hline $3.77 \mathrm{e}-03$ & 3.84 & $7.17 \mathrm{e}-04$ & 3.98 & 15 & 15 & $1.79 \mathrm{e}+04$ \\
\hline $9.71 \mathrm{e}-04$ & 3.88 & $7.77 \mathrm{e}-05$ & 9.22 & 31 & 31 & $2.03 \mathrm{e}+05$ \\
\hline $2.32 \mathrm{e}-04$ & 4.18 & $7.23 \mathrm{e}-06$ & 10.7 & 63 & 63 & $1.62 \mathrm{e}+06$ \\
\hline $6.08 \mathrm{e}-05$ & 3.82 & $5.45 \mathrm{e}-07$ & 13.8 & 127 & 127 & $8.78 \mathrm{e}+06$ \\
\hline $1.51 \mathrm{e}-05$ & 4.03 & $3.72 \mathrm{e}-08$ & 14.6 & 255 & 255 & $3.86 \mathrm{e}+07$ \\
\hline
\end{tabular}

Table 4.2: Simulations with Linear/Cubic method for beam (no patch)

The results in Table 4.2 show that quadratic convergence rate (halving the mesh size, equivalently, doubling the number of basis elements, produces one quarter the error) and quartic convergence rate (halving the mesh size produces one sixteenth the error) are observed for $v_{c}$ and $w_{c}$ in Test 1 and Test 2, respectively. In Test 3, the highest rate obtained, 14.6 , for the $w_{c}$ component is not observed until $N=(255,255)$. This justifies our early conjecture that the coupling with the $v_{c}$ component, which has a slower convergence rate, causes a slowed rate in $w_{c}$.

Simulation 2. Next, we apply the Cubic/Cubic method for Test 3 (Table 4.3).

The results in Table 4.3 show that even with coupling, the quartic convergence rates for both $v_{c}$ and $w_{c}$ in this case, are observed at the approximation level $N=$ $(33,31)$. Furthermore, there is not much fluctuation in the rates of convergence after this level of discretization.

Simulation 3. Now we include one pair of patches positioned over the interval $[.25, .5]$ on the beam, and we use the Linear/Cubic method for Tests 1-3 (Table 4.4).

First note that it takes much finer meshes, hence more basis elements, to obtain the expected convergence rates for all the tests in this simulation. Physically, the patched model has jumps in its thickness, and mathematically, the patches introduce discon- 


\begin{tabular}{r||r|c||r|c|r|r|r||}
\hline \multirow{5}{*}{} & Verr & ratio of error & Werr & ratio of error & $N_{v}$ & $N_{w}$ & $K$-cond \\
\hline \multirow{5}{*}{ Test 3: } & $7.19 \mathrm{e}-03$ & & $5.61 \mathrm{e}-03$ & & 5 & 3 & $9.39 \mathrm{e}+02$ \\
\cline { 2 - 8 } & $6.26 \mathrm{e}-04$ & 11.5 & $3.69 \mathrm{e}-04$ & 15.2 & 9 & 7 & $2.78 \mathrm{e}+04$ \\
\cline { 2 - 8 } & $1.94 \mathrm{e}-05$ & 32.2 & $1.53 \mathrm{e}-05$ & 24.1 & 17 & 15 & $1.01 \mathrm{e}+05$ \\
\cline { 2 - 8 } & $1.13 \mathrm{e}-06$ & 17.3 & $9.38 \mathrm{e}-07$ & 16.3 & 33 & 31 & $3.86 \mathrm{e}+05$ \\
\hline & $7.00 \mathrm{e}-08$ & 16.1 & $5.45 \mathrm{e}-08$ & 17.2 & 65 & 63 & $1.53 \mathrm{e}+06$ \\
\hline
\end{tabular}

Table 4.3: Simulations with Cubic/Cubic method for beam (no patch)

Test 1:

\begin{tabular}{||r|c|r|r|c||}
\hline Verr & ratio of error & $N_{v}$ & $N_{w}$ & $K$-cond \\
\hline $7.63 \mathrm{e}-02$ & & 3 & 3 & $1.20 \mathrm{e}+02$ \\
\hline $4.06 \mathrm{e}-02$ & 1.88 & 7 & 3 & $1.07 \mathrm{e}+03$ \\
\hline $2.31 \mathrm{e}-02$ & 1.76 & 15 & 3 & $6.30 \mathrm{e}+03$ \\
\hline $9.63 \mathrm{e}-03$ & 2.40 & 31 & 3 & $3.63 \mathrm{e}+04$ \\
\hline $2.97 \mathrm{e}-03$ & 3.25 & 63 & 3 & $1.66 \mathrm{e}+05$ \\
\hline $8.01 \mathrm{e}-04$ & 3.63 & 127 & 3 & $6.67 \mathrm{e}+05$ \\
\hline $2.02 \mathrm{e}-04$ & 3.97 & 255 & 3 & $2.61 \mathrm{e}+06$ \\
\hline
\end{tabular}

\begin{tabular}{||r||c|c|r|r|c||}
\hline \multicolumn{1}{c||}{} & Werr & ratio of error & $N_{v}$ & $N_{w}$ & $K$-cond \\
\hline $3.17 \mathrm{e}-03$ & & 3 & 3 & 120 \\
\hline \multirow{3}{*}{ Test 2: } & $2.18 \mathrm{e}-04$ & 14.5 & 3 & 7 & $2.78 \mathrm{e}+02$ \\
\cline { 2 - 6 } & $1.37 \mathrm{e}-05$ & 15.9 & 3 & 15 & $3.73 \mathrm{e}+02$ \\
\cline { 2 - 6 } & $9.11 \mathrm{e}-07$ & 15.0 & 3 & 31 & $7.17 \mathrm{e}+02$ \\
\cline { 2 - 6 } & $4.77 \mathrm{e}-08$ & 19.1 & 3 & 63 & $5.63 \mathrm{e}+03$ \\
\hline & $2.94 \mathrm{e}-09$ & 16.2 & 3 & 127 & $8.93 \mathrm{e}+04$ \\
\hline & $1.91 \mathrm{e}-10$ & 15.4 & 3 & 255 & $1.42 \mathrm{e}+06$ \\
\hline
\end{tabular}

\begin{tabular}{|c|c|c|c|c|c|c|c|}
\hline \multirow{8}{*}{ Test 3: } & Verr & ratio of error & Werr & ratio of error & $N_{v}$ & $N_{w}$ & $K$-cond \\
\hline & $7.47 \mathrm{e}-02$ & & $9.04 \mathrm{e}-03$ & & 3 & 3 & $1.20 \mathrm{e}+02$ \\
\hline & $3.93 \mathrm{e}-02$ & 1.90 & $8.61 \mathrm{e}-03$ & 1.05 & 7 & 7 & $1.33 \mathrm{e}+03$ \\
\hline & $3.16 \mathrm{e}-02$ & 1.24 & $9.02 \mathrm{e}-03$ & 0.954 & 15 & 15 & $1.92 \mathrm{e}+04$ \\
\hline & $2.16 \mathrm{e}-02$ & 0.955 & $6.57 \mathrm{e}-03$ & 0.943 & 31 & 31 & $1.30 \mathrm{e}+05$ \\
\hline & $9.72 \mathrm{e}-03$ & 2.23 & $2.97 \mathrm{e}-03$ & 2.21 & 63 & 63 & $2.07 e+05$ \\
\hline & $3.09 \mathrm{e}-03$ & 3.14 & $9.38 \mathrm{e}-04$ & 3.17 & 127 & 127 & $1.47 \mathrm{e}+06$ \\
\hline & $8.27 \mathrm{e}-04$ & 3.74 & $2.52 \mathrm{e}-04$ & 3.72 & 255 & 255 & $9.58 \mathrm{e}+07$ \\
\hline
\end{tabular}

Table 4.4: Simulations with Linear/Cubic method for patched beam 
tinuities in the parameters such as $\bar{\rho}, E, C_{D}$. We observe that this nonsmoothness requires larger values of $N$ (i.e., a finer discretization) to achieve good approximations. Consequently, for the same level of accuracy (the relative errors), the condition numbers for $K$ in Test 3 have become larger than those from Test 3 of Simulation 1 for the beam-without-patch model.

In Test 1 (Table 4.4), quadratic convergence rate is not observed until 255 linear splines (compared with 15 in Test 1 of Simulation 1 (Table 4.2)) are used to approximate the decoupled $V^{*}$. (Experiments with higher values for $N_{w}$ produce similar rates). As a consequence, this delay triggers another delay in reaching a convergence rate between quadratic and quartic for the $w_{c}$ component in Test 3 at an acceptable stage. On the other hand, the errors from Test 2 (Table 4.4), when $V^{*}=0$, produce quartic rate at a comparable stage to that in Test 2 of Simulation 1. This prompts our next simulation.

Simulation 4. We use the Cubic/Cubic method for Test 3 (Table 4.5).

Test 3:

\begin{tabular}{||r|c||r|c|r|r|c||}
\hline Verr & ratio of error & Werr & ratio of error & $N_{\vartheta}$ & $N_{w}$ & $K$-cond \\
\hline $7.11 \mathrm{e}-03$ & & $5.12 \mathrm{e}-03$ & & 5 & 3 & $6.08 \mathrm{e}+02$ \\
\hline $8.39 \mathrm{e}-04$ & 8.47 & $4.37 \mathrm{e}-04$ & 11.7 & 9 & 7 & $1.03 \mathrm{e}+04$ \\
\hline $2.08 \mathrm{e}-05$ & 40.3 & $1.57 \mathrm{e}-05$ & 27.9 & 17 & 15 & $5.65 \mathrm{e}+04$ \\
\hline $1.19 \mathrm{e}-06$ & 17.5 & $9.47 \mathrm{e}-07$ & 16.5 & 33 & 31 & $2.44 \mathrm{e}+05$ \\
\hline $7.38 \mathrm{e}-08$ & 16.1 & $5.47 \mathrm{e}-08$ & 17.3 & 65 & 63 & $1.02 \mathrm{e}+06$ \\
\hline $4.59 \mathrm{e}-09$ & 16.1 & $3.47 \mathrm{e}-09$ & 15.8 & 129 & 127 & $5.22 \mathrm{e}+06$ \\
\hline
\end{tabular}

Table 4.5: Simulations with Cubic/Cubic method for patched beam

The results in Table 4.5 show that we observe quartic convergence rate at the same stage as that in Test 3 of Simulation 2 (Table 4.3) for the beam-without-patch model. Considering the basis elements, we find that if the linear B-spline elements are used in the model, their first derivatives, which are not continuous, also appear. On the other hand, the cubic splines have both continuous first and second derivatives. We conjecture this is one reason that the effect of including patches on the Cubic/Cubic method is not as significant as it is on the Linear/Cubic method.

To summarize our results for this subsection, we conclude that we do observe the expected convergence rates in all the above simulations. We also observe that the relative errors associated with the Cubic/Cubic method are significantly smaller than those with the Linear/Cubic method for the same mesh. With one pair of patches in the model, the Linear/Cubic method takes as many as thirty-two times of the number of basis elements used in the Cubic/Cubic method to reach the same order of accuracy, referring to level $N=(255,255)$ of Test 3 in Simulation 3 (Table 4.4) and level $N=(9,7)$ of Test 3 in Simulation 4 (Table 4.5). However, the condition numbers of the matrix $K$ from the Cubic/Cubic method are somewhat larger than 
those from the Linear/Cubic method.

\subsubsection{Dynamic case}

The set of true solutions are chosen to be

$$
\begin{gathered}
V^{*}=v(t, \theta)=\left(-0.4 \theta^{2}+0.4 \theta\right) t^{2} \\
W^{*}=w(t, \theta)=\left(16 \theta^{4}-32 \theta^{3}+16 \theta^{2}\right) t^{2}
\end{gathered}
$$

where $0 \leq t \leq 1.0$. The measurement comparisons are done at $t=1.0$, hence the relative errors are Verr $=\left\|V^{*}(1, \cdot)-v_{c}(1, \cdot)\right\| /\left\|V^{*}(1, \cdot)\right\|$ and Werr $=\| W^{*}(1, \cdot)-$ $w_{c}(1, \cdot)\|/\| W^{*}(1, \cdot) \|$. The norm is again the sup norm over $\theta \in(0,1)$. For the following runs, we also look at CPU time, the total amount of time used to solve (3.6).

Simulation 5. First, we assume there are no patches bonded to the beam and apply the Linear/Cubic method for Test 3 (Table 4.6).

Test 3:

\begin{tabular}{||r|c||r|c|r|r|c||}
\hline Verr & ratio of error & Werr & ratio of error & $N_{v}$ & $N_{w}$ & CPU(sec) \\
\hline $6.05 \mathrm{e}-03$ & & $6.69 \mathrm{e}-03$ & & 3 & 3 & 10 \\
\hline $1.44 \mathrm{e}-03$ & 4.21 & $2.83 \mathrm{e}-03$ & 2.36 & 7 & 7 & 46 \\
\hline $3.75 \mathrm{e}-04$ & 3.84 & $7.21 \mathrm{e}-04$ & 3.93 & 15 & 15 & 278 \\
\hline $9.66 \mathrm{e}-05$ & 3.88 & $7.79 \mathrm{e}-05$ & 9.26 & 31 & 31 & 2189 \\
\hline $2.39 \mathrm{e}-05$ & 4.05 & $7.21 \mathrm{e}-06$ & 10.8 & 63 & 63 & 17362 \\
\hline
\end{tabular}

Table 4.6: Simulations (dynamic) with Linear/Cubic method for beam (no patch)

Simulation 6. We apply the Cubic/Cubic method for Test 3 (Table 4.7).

Test 3:

\begin{tabular}{||r|c||r|c|r|r|c||}
\hline Verr & ratio of error & Werr & ratio of error & $N_{v}$ & $N_{w}$ & CPU(sec) \\
\hline $7.19 \mathrm{e}-04$ & & $5.60 \mathrm{e}-03$ & & 5 & 3 & 14 \\
\hline $6.26 \mathrm{e}-05$ & 11.5 & $3.69 \mathrm{e}-04$ & 15.2 & 9 & 7 & 52 \\
\hline $1.94 \mathrm{e}-06$ & 32.2 & $1.53 \mathrm{e}-05$ & 24.1 & 17 & 15 & 314 \\
\hline $1.13 \mathrm{e}-07$ & 17.3 & $9.38 \mathrm{e}-07$ & 16.3 & 33 & 31 & 2664 \\
\hline
\end{tabular}

Table 4.7: Simulations (dynamic) with Cubic/Cubic method for beam (no patch)

As we have observed in the Simulations 1 and 2 of the static case, we obtain expected convergence rates in both $v$ and $w$.

Simulation \%. Now we include one pair of patches positioned over the interval $[.25, .5]$ on the beam. We use Cubic/Cubic method with Test 3 (Table 4.8).

We conclude that we observe the expected convergence rates in Simulations 5-7. The results from the dynamic model confirm that the Cubic/Cubic method is more 


\begin{tabular}{||r||r|c||r|r|r|r|c||}
\hline \multicolumn{1}{c||}{} & Verr & ratio of error & Werr & ratio of error & $N_{v}$ & $N_{w}$ & CPU(sec) \\
\hline \multirow{5}{*}{ Test 3: } & $7.12 \mathrm{e}-04$ & & $5.11 \mathrm{e}-03$ & & 5 & 3 & 15 \\
\cline { 3 - 10 } & $8.39 \mathrm{e}-05$ & 8.48 & $4.36 \mathrm{e}-04$ & 11.7 & 9 & 7 & 71 \\
\cline { 2 - 9 } & $2.09 \mathrm{e}-06$ & 40.2 & $1.57 \mathrm{e}-05$ & 27.7 & 17 & 15 & 443 \\
\cline { 2 - 8 } & $1.19 \mathrm{e}-07$ & 17.5 & $9.69 \mathrm{e}-07$ & 16.2 & 33 & 31 & 4022 \\
\hline & $7.68 \mathrm{e}-09$ & 15.5 & $6.29 \mathrm{e}-08$ & 15.4 & 65 & 63 & 26146 \\
\hline
\end{tabular}

Table 4.8: Simulations (dynamic) with Cubic/Cubic method for patched beam

accurate for the same mesh, hence that it is also more time-efficient than the Linear/Cubic method. (In the remainder of the numerical experiments, the computations are carried out with the Cubic/Cubic method if not indicated otherwise).

\subsection{Modes and Mode Shapes}

In this section, we continue the study of the dynamic behavior of the curved beam (without patches vs. with patches) by first evaluating its fundamental frequencies and mode shapes, which are very important in engineering studies. One way to obtain approximate frequencies and mode shapes is by evaluating the eigenvalues and the eigenvectors of the unforced and undamped approximate system

$$
M \ddot{z}+K z=0
$$

for sufficiently large values of the approximation indices $N=\left(N_{v}, N_{w}\right)$. The associated eigen-problem can be written as

$$
\omega^{2} M X=K X,
$$

by making the usual analogy to the one-dimensional spring-mass problem, in which $M, K, z$ are just scalars. Since this is a multidimensional problem, there are $N_{v}+N_{w}$ eigenvalues, and hence the same number of fundamental frequencies for the approximate system. Each $\omega_{i}$ is an angular frequency, while the natural frequency (in hertz) can be obtained by $f_{i}=\omega_{i} /(2 \pi)$. The corresponding eigenvectors $X$ can be used along with the basis elements to compute the approximate mode shapes.

We tabulate in Table 4.9 the first four natural frequencies of the curved beam without patches. We used $N=(17,15)$ in this simulation since calculations with other choices of $N=\left(N_{v}, N_{w}\right)$ suggested that the above frequencies yielded reasonable approximation to those of the system. The corresponding mode shapes are given in Figure 4.1. 


\begin{tabular}{||c|c|c|c||l|l||}
\hline$f_{1}(\mathrm{hz})$ & $f_{2}(\mathrm{hz})$ & $f_{3}(\mathrm{hz})$ & $f_{4}(\mathrm{hz})$ & $N_{v}$ & $N_{w}$ \\
\hline 101 & 190 & 342 & 502 & 17 & 15 \\
\hline
\end{tabular}

Table 4.9: Fundamental frequencies of the beam (no patch)
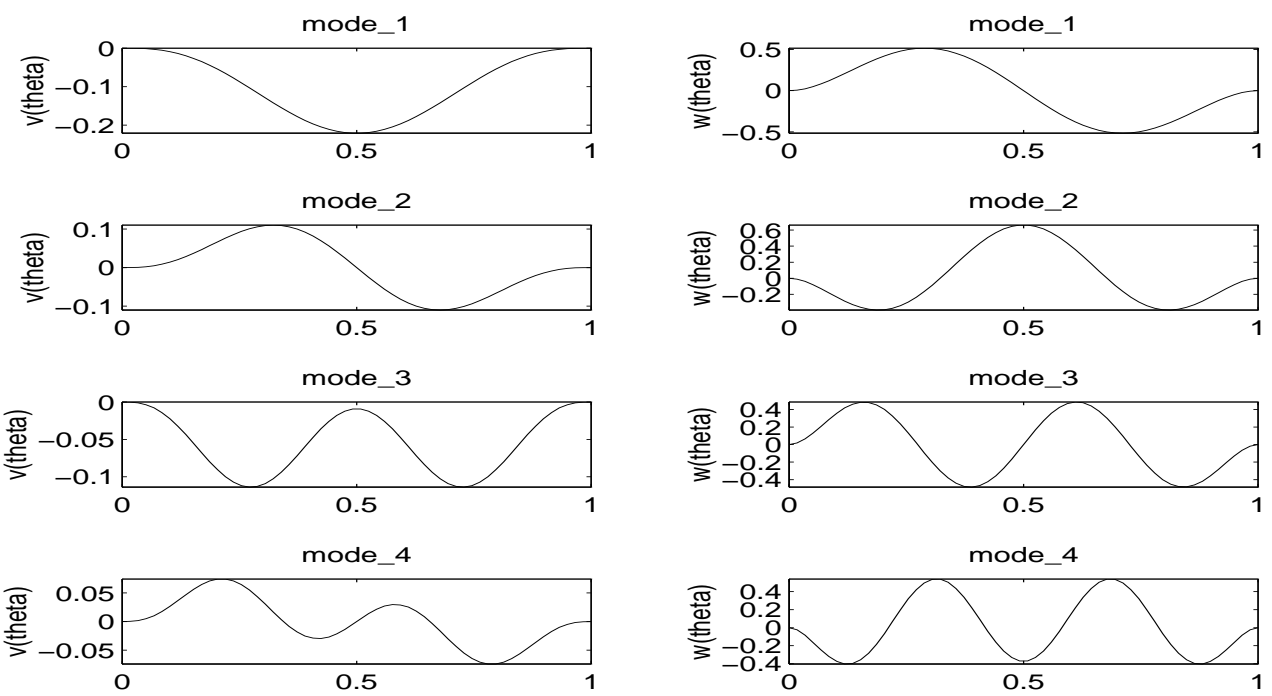

Figure 4.1 Mode shapes for curved beam (no patch)

In Figure 4.1, the horizontal axes are $\theta \in[0,1]$. Each row is one mode shape which contains a $v$ component and a $w$ component of the system vibrations. The $v$ component and the $w$ component do not exist independently, so it is somewhat misleading (at the least) to define them as extensional modes and bending modes. This would be correct terminology if the coupling terms in the model were not present. However, the uncoupled problem would have the first few fundamental frequencies as $548 \mathrm{hz}$ and $1095 \mathrm{hz}$ for the extensional motions and $2151 \mathrm{hz}$ and $2171 \mathrm{hz}$ for the bending motions.

\begin{tabular}{||c|c|c|c||l|l||}
\hline $\mathrm{f} 1(\mathrm{hz})$ & $\mathrm{f} 2(\mathrm{hz})$ & $\mathrm{f} 3(\mathrm{hz})$ & $\mathrm{f} 4(\mathrm{hz})$ & $N_{v}$ & $N_{w}$ \\
\hline 117 & 195 & 454 & 620 & 65 & 63 \\
\hline
\end{tabular}

Table 4.10: Fundamental frequencies of patched beam

Next, we include one pair of patches positioned over the interval $[.25, .5]$ on the beam and tabulate in Table 4.10 the first four natural frequencies. Again, the specific choice of $N=(65,63)$ is based on comparisons with results from calculations with 
different $N_{s}$. Note the rather significant fundamental frequency changes from those of the beam with no patch: we observe around $15 \%$ shift at low frequencies and $20 \%$ shift at higher frequencies. Moreover, this occurs simply from adding the passive patches which load the beam with respect to mass (slightly) and stiffness (significantly). We obtain some implications that are important to engineering studies - we cannot ignore patch loading, just as we cannot calculate separate frequencies for extensional and bending vibrations and then combine them.

The first four mode shapes of the patched beam are:
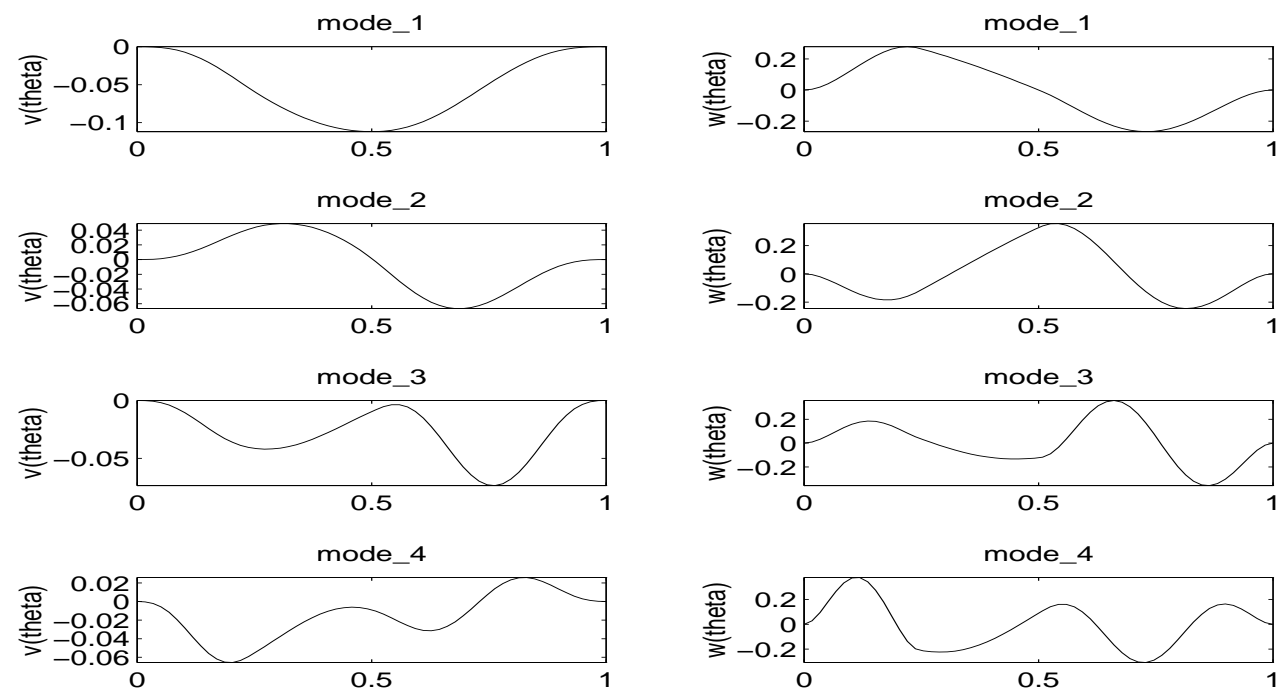

Figure 4.2 Mode shapes for patched curved beam

Again in Figure 4.2, each row is one mode shape with a $v$-component and a $w$ component. The first two mode shapes remain similar to those of the beam-withoutpatch model. However, all the mode shapes are no longer even or odd about the middle of the beam (as expected since the patches are bonded off-center on the beam).

\subsection{Forced vibration}

\subsubsection{Hammer hit}

As indicated in the previous subsection, another way to obtain the fundamental frequencies is by applying an external force that excites all of them and then by applying FFT to the solutions in time, $v\left(t, \theta_{0}\right)$ and $w\left(t, \theta_{0}\right)$, where $\theta_{0}$ is some point on the beam. We used a triangular forcing function to simulate a hammer hit input, which is commonly used in laboratories.

$$
f(t)=\left\{\begin{array}{ll}
10000 t & 0 \leq t \leq 1 / 1024 \\
-10000 t+(2 / 1024) 10000 & 1 / 1024<t \leq 2 / 1024
\end{array} .\right.
$$


The external force vector $F$ defined in (3.5) is then taken to be

$$
F=f(t) \int_{0}^{1}\left(\phi_{1}, \phi_{2} \cdots \phi_{N_{v}}, \psi_{1}, \psi_{2} \cdots \psi_{N_{w}}\right)^{T} d \theta .
$$

In the simulations of this section, the mesh with $N=(17,15)$ cubic/cubic splines is applied when a beam without patches is considered, and the mesh with $N=(65,63)$ cubic/cubic splines is applied when a patched beam is considered. In each of the following figure, results for $v\left(t, \theta_{0}\right)$ are displayed before the results for $w\left(t, \theta_{0}\right)$.

Simulation 1. First we assume no patches on the beam and no damping in the model, which becomes $M \ddot{z}+K z=F$.
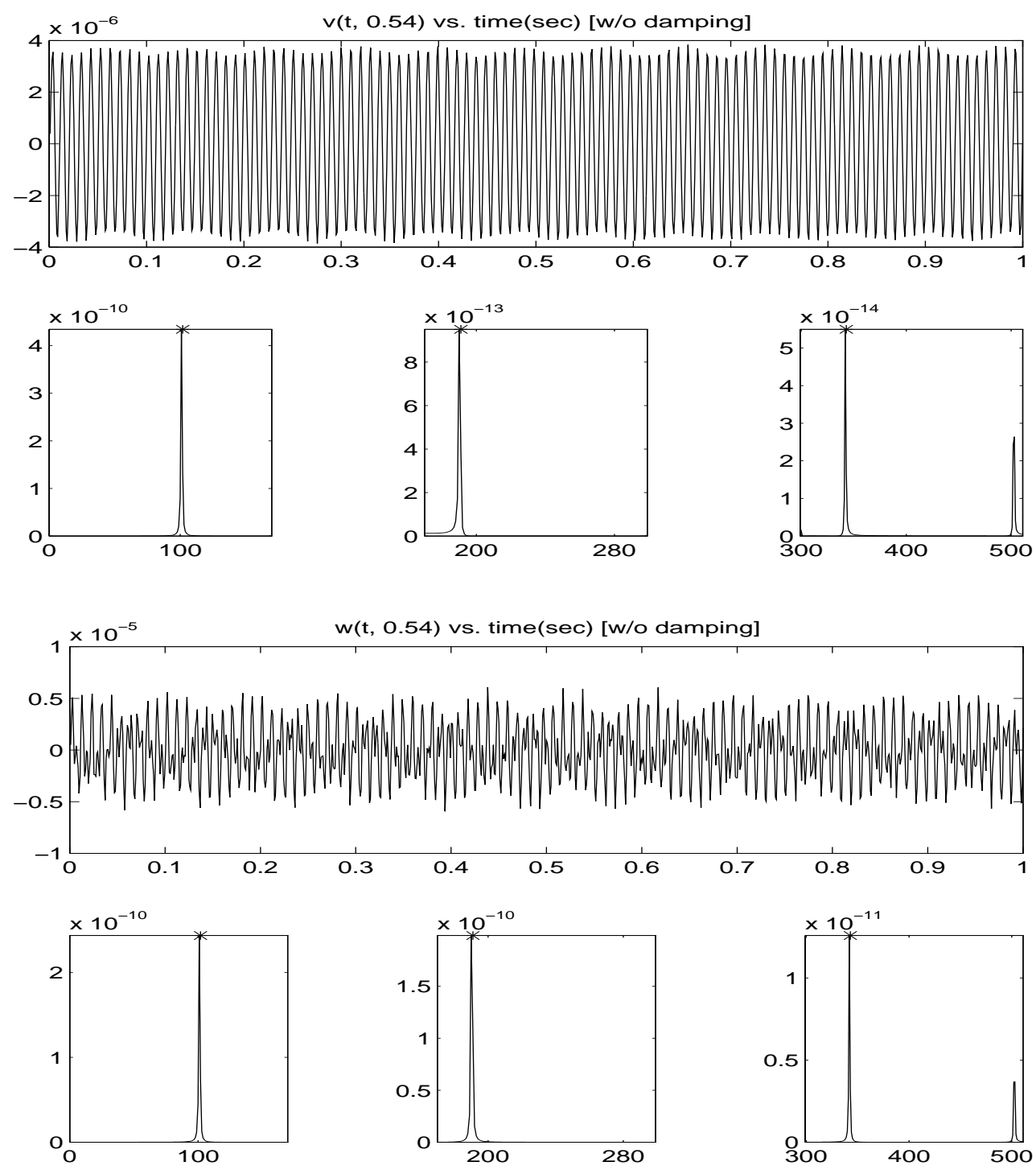

Figure 4.3 FFT for beam (no patch) w/o damping 
The frequency readings from the $v$ plots are 102, 191, 343, $504 \mathrm{hz}$ (second row in the above figure), and from the $w$ plots are 102, 191, 343, $503 \mathrm{hz}$ (fourth row). We conclude that the readings from the power spectra match the results obtained from the eigen-problem within the resolution of the frequency axis, which is $\Delta f=1 \mathrm{hz}$.

Simulation 2. We include (internal) Kelvin-Voigt damping in the model, hence the damping matrix $C$ is calculated and the model becomes (3.5). In addition, we include (external) air damping only in the radial direction; that is, we add $-\int_{0}^{1} \gamma \dot{W}^{*} \psi_{k} d \theta, 1 \leq$ $k \leq N_{w}$ to the last $N_{w}$ entries of $F$. (The effective area of air damping in the tangential direction was taken to be negligible). This provides a test in a practical setting where both internal and external damping are present.
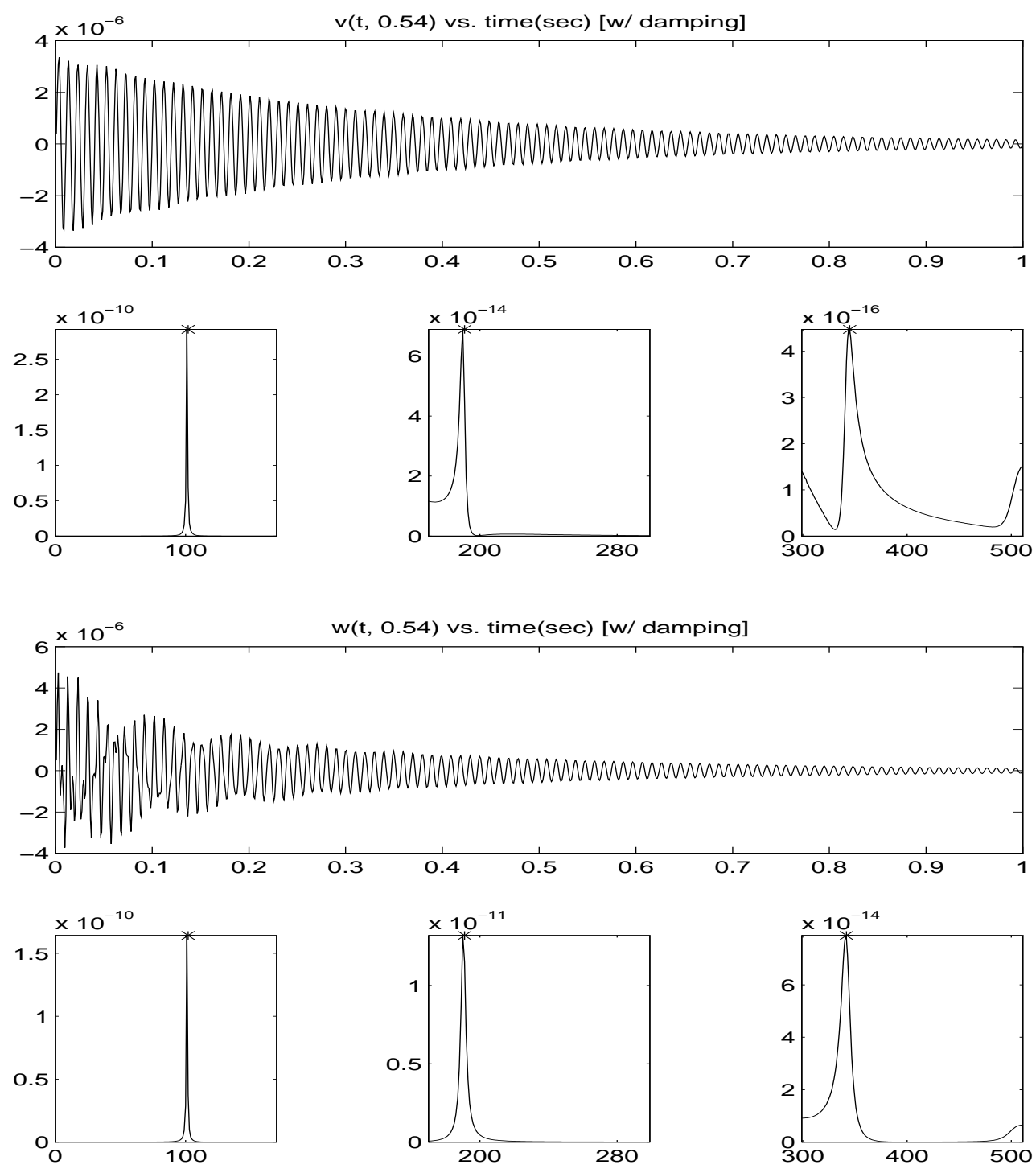

Figure 4.4 FFT for beam (no patch) w/ damping 
The frequency readings from the $v$ plots are $102,191,345 \mathrm{hz}$, and from the $w$ plots are 102, 191, 342 hz. Comparing Figure 4.4 to Figure 4.3, we can conclude that the inclusion of the Kelvin-Voigt damping and air-damping is responsible for the slight decrease of the magnitudes of the power densities and the "disappearing" of the fourth mode (i.e., it is greatly damped and it is not observed with this excitation input).

Simulation 3. Next we include one pair of patches positioned over the interval $[.25, .5]$ on the beam, and we assume at first that there is no damping:
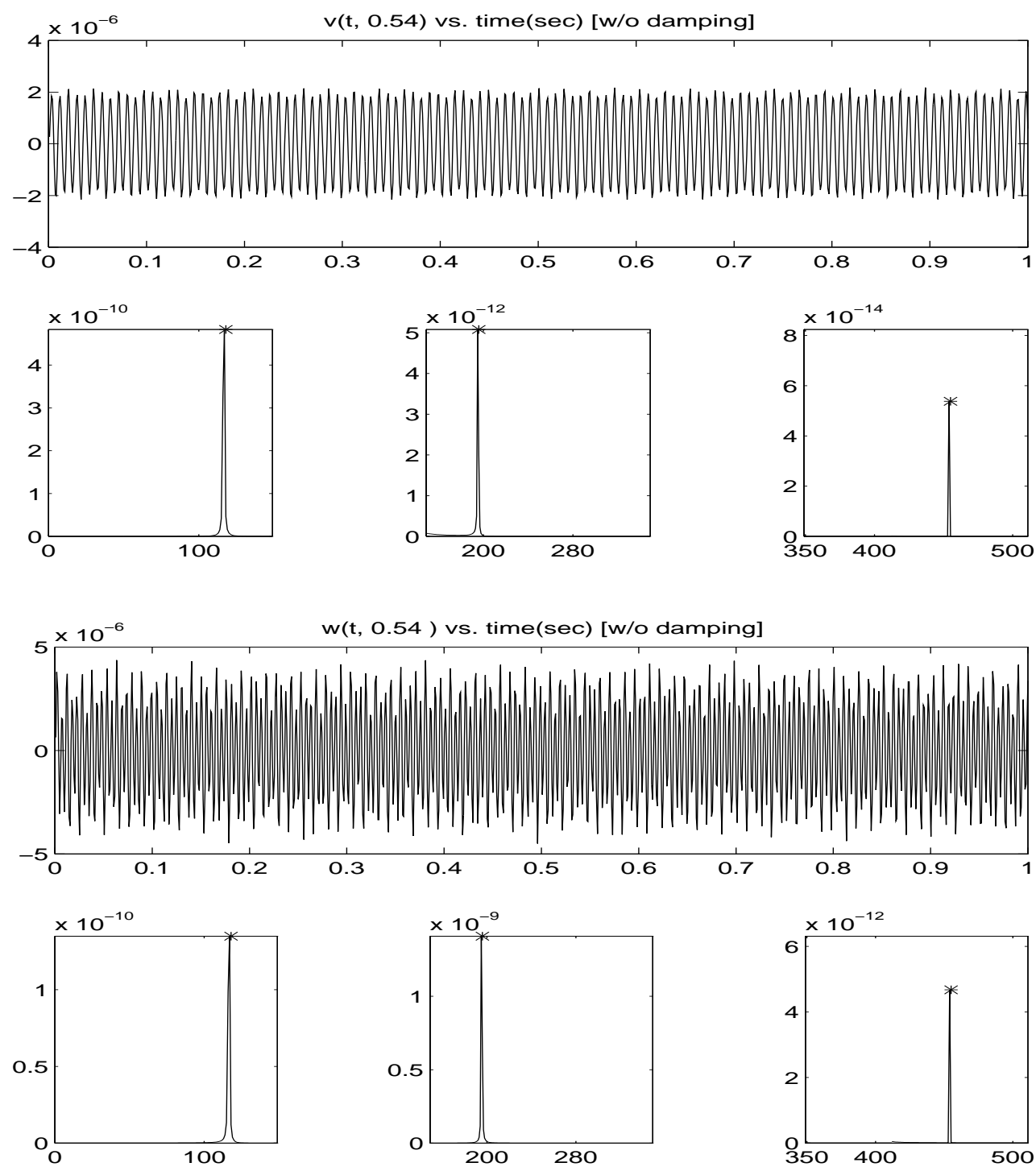

Figure 4.5 FFT for patched beam w/o damping

The frequency readings from both the $v$ plots and the $w$ plots are $118,196,455 \mathrm{hz}$, 
matching the results from the eigen problem within the resolution of the frequency axis.

Simulation 4. We include Kelvin-Voigt damping in both directions of the vibrations and air damping only in the radial direction for the patched beam.
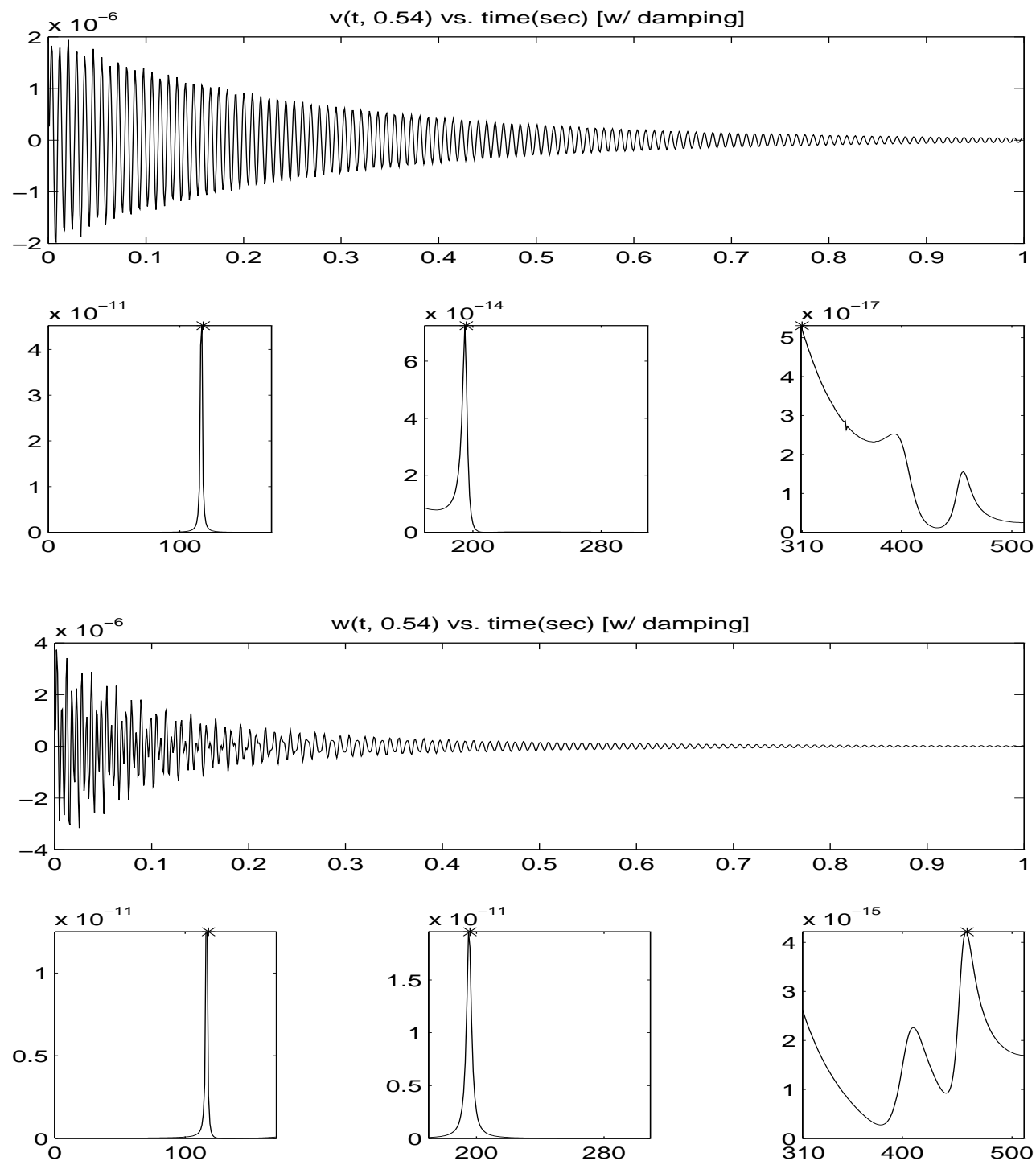

Figure 4.6 FFT for patched beam w/ damping

The frequency readings from the $v$ plots and the $w$ plots are 118 and $196 \mathrm{hz}$. We observe that the third mode and the fourth mode have been eliminated (i.e., they are greatly damped and they are not observed with this excitation input).

We conclude for this subsection that the frequencies, of the beam and of the beam with patches, excited by the hammer hit match the results from the corresponding 
eigen problems. The frequencies of the patched beam are higher than those of the beam alone, and the mode shapes show a less regular pattern than those of the unpatched beam.

\subsubsection{Activated patches}

In this experiment, we apply voltages to the PZT piezoceramic patches following the rule that the maximum voltages to be applied to a patch is $8-10 \mathrm{rms} V / \mathrm{mil}$ (see [BS]). For the patches used in our model, the maximum rms $V$ is around 75 Volts, and we choose the maximum amplitude to be 7 Volts. The following voltage functions are applied to patch 1 and 2 to activate vibrations in the structure:

$$
V_{1}=-V_{2}=7 \sin (240 \pi t)
$$
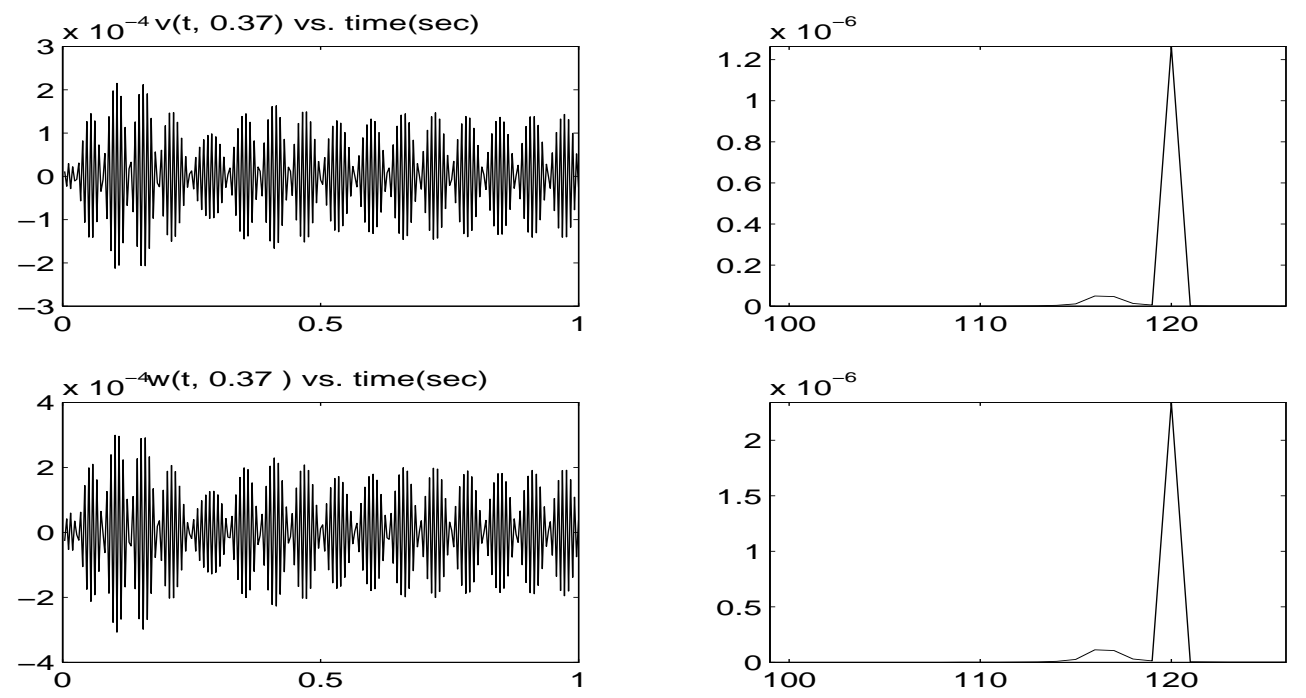

Figure 4.7 Patch activated beam w/ damping

The frequency readings of the two peaks in the power spectra are 118, $121 \mathrm{hz}$, as we expected. The former is the fundamental frequency of the patched beam, and the latter is the driving frequency. Furthermore, in the displacement plots, the beat phenomenon which occurs in systems near resonance is also manifested.

Since $V_{1}=-V_{2}$ in this case, the discussion in Section 2.2.2 implies that there is no extensional force, $\hat{q}_{\theta}$, on the beam. That is, there is no external agent driving the structure in the tangential direction, yet the magnitudes of $v(t, .37)$ are as significant as those of $w(t, .37)$ due to the coupling of the two components. This observation affirms the effects of the coupling terms in the model. 


\section{$5 \quad$ Locking}

In this section, we discuss a topic that has received growing attention over the last two decades. In some shell and plate models, e.g., the Reissner-Mindlin plate model, a phenomenon associated with numerical over stiffness of the approximation scheme has been classified as locking. It is described as:

"A numerical scheme for the approximation of a parameter-dependent problem is said to exhibit locking if the accuracy of the approximation deteriorates as the parameter tends to a limiting value" (see [BS1]).

We refer the readers to $[\mathrm{A}]$, [SBS] and [Z] for a more detailed definition and for the definition of the order of locking. To explain the occurrence of locking, one investigates constraints that appear as certain parameters in the physical problems tend to limiting values. These constraints are called "hidden" in [LP]. They usually introduce extra conditions that are difficult to satisfy or even approximate well in the numerical computations with the models. For example, the constraint that produces shear locking in the Reissner-Mindlin plate model results from enforcing the Kirchhoff hypothesis when the thickness gets small (see $[A F]$ ), and the constraints that produce membrane locking in the bending dominated hemishell problem described in [LP] result from enforcing inextensional theory which must hold in the limit as the thickness gets small.

As our curved beam model is also a parameter dependent problem, we examine the model (static case with no patches) by decreasing its thickness, $h$, and study the relative errors defined in Section 4.1.1. We present two cases of the model in which the external force is respectively parallel to one of the displacement directions, and in which we observe locking-type phenomena.

Case 1. We assume that the only nonzero external (line) force is tangential with magnitude $b \hat{q}_{\theta}=(4 e+6) h^{3}$. This is in an attempt to provide an analog in our 1-D curvature model to the Reissner-Mindlin plate discussed in [AF] and [SBS]

$$
\begin{aligned}
\frac{-E h b}{R^{2}} \partial(\partial v+w) & =b \hat{q}_{\theta} \\
\frac{E h^{3} b}{12 R^{4}} \partial^{2}\left(\partial^{2} w\right)+\frac{E h b}{R^{2}}(\partial v+w) & =0 .
\end{aligned}
$$

The classical solutions (from using Maple), $V^{*}$ and $W^{*}$, are fifth degree and sixth degree polynomials, respectively. By taking $h \rightarrow 0$ in (5.2), we obtain $\partial v+w=0$, which is sometimes referred to as the "Love assumption" and arises in the (2-dimensional) inextensional theory (see [A1]). This result becomes a constraint that is very difficult for low order basis functions to satisfy in the Galerkin finite element approximation scheme. For instance, with the Linear/Cubic method, piecewise linear functions are used to approximate $V^{*}$ and cubic B-spline functions are used to approximate $W^{*}$. The constraint requires a finite sum of the derivatives, which are piecewise constants, 
of the piecewise linear functions, to be a cubic function. Similarly, with the $\mathrm{Cu}^{-}$ bic/Cubic method, the constraint requires compatibility between piecewise quadratic functions and cubic functions, which in this case represents a weaker condition of compatibility. We compared the two schemes for several values of decreasing thickness $h$.

Linear/Cubic method

\begin{tabular}{||c|c|c||l|l||}
\hline thickness & Verr & Werr & $N_{v}$ & $N_{w}$ \\
\hline \multirow{3}{*}{$5.2 \mathrm{e}-3$} & .19 & .20 & 127 & 7 \\
\cline { 2 - 5 } & .06 & .06 & 255 & 7 \\
\hline \multirow{3}{*}{$5.2 \mathrm{e}-4$} & .85 & .85 & 255 & 7 \\
\cline { 2 - 5 } & .60 & .60 & 511 & 7 \\
\cline { 2 - 5 } & .27 & .27 & 1023 & 7 \\
\hline $5.2 \mathrm{e}-5$ & 1.0 & 1.0 & 511 & 7 \\
\cline { 2 - 5 } & 1.0 & 1.0 & 1023 & 7 \\
\hline
\end{tabular}

Table 5.1: Simulation (tangential load) results with Linear/Cubic method for beam with decreasing thickness

Cubic/Cubic method

\begin{tabular}{||c|c|c||l|l||}
\hline thickness & Verr & Werr & $N_{v}$ & $N_{w}$ \\
\hline $5.2 \mathrm{e}-3$ & .9 & .9 & 5 & 3 \\
\cline { 2 - 5 } & .06 & .06 & 9 & 7 \\
\cline { 2 - 5 } & .001 & .001 & 17 & 15 \\
\hline \multirow{5}{*}{$5.2 \mathrm{e}-5$} & .9 & .9 & 17 & 15 \\
\cline { 2 - 5 } & .12 & .12 & 33 & 31 \\
\cline { 2 - 5 } & .002 & .002 & 65 & 63 \\
\hline $5.2 \mathrm{e}-7$ & 1.0 & 1.0 & 65 & 63 \\
\cline { 2 - 5 } & 1.0 & 1.0 & 129 & 127 \\
\cline { 2 - 5 } & 1.0 & 1.0 & 513 & 511 \\
\hline
\end{tabular}

Table 5.2: Simulation (tangential load) results with Cubic/Cubic method for beam with decreasing thickness

For each value of $h$, the mesh refinement iterations were terminated when the magnitude of the condition number of the corresponding stiffness matrix, $K$ (defined in Section 4.1.1.), exceeded $10^{10}$. We observe that when using Linear/Cubic method (calculations with $N_{w}=15$ produced similar results to those shown in Table 5.1), even with the finest acceptable mesh, the relative errors are still large for the beam 
with thickness $h=5.2 e-4$. When the Cubic/Cubic method (Table 5.2) is used, a dramatic improvement in accuracy is noted and $h$ can be reduced further. However, the results for the beam with $h=5.2 e-7$ are not yet converged with the finest acceptable mesh.

Based on our understanding of the locking phenomenon, we conclude that the above observation certainly fits the definition of locking in the beginning of this section and may be classified as curvature locking (also referred to as membrane locking, see [LP]) since the constraint is due to the curvature in the physical model. As expected in such situations, we suggest that finer meshes and special algorithms (e.g., see [Z]) can overcome the difficulties that are observed here as $h \rightarrow 0$.

Case 2. In this case, we assume that the only nonzero external (line) force is $b \hat{q}_{n}$ and that it is a constant function in $h$, e.g., $b \hat{q}_{n}=4$ :

$$
\begin{aligned}
\frac{-E h b}{R^{2}} \partial(\partial v+w) & =0 \\
\frac{E h^{3} b}{12 R^{4}} \partial^{2}\left(\partial^{2} w\right)+\frac{E h b}{R^{2}}(\partial v+w) & =b \hat{q}_{n} .
\end{aligned}
$$

This model represents not only a common occurrence in practice but is also a dual case to the previous one. The classical solutions, $V^{*}$ and $W^{*}$, are fifth degree and fourth degree polynomials. By taking $h \rightarrow 0$, we obtain $\partial v+w=$ constant, which is not a particular constraint on low order basis functions. However, the results from using the Linear/Cubic method manifest a locking type of behavior as seen in Figure 5.1.
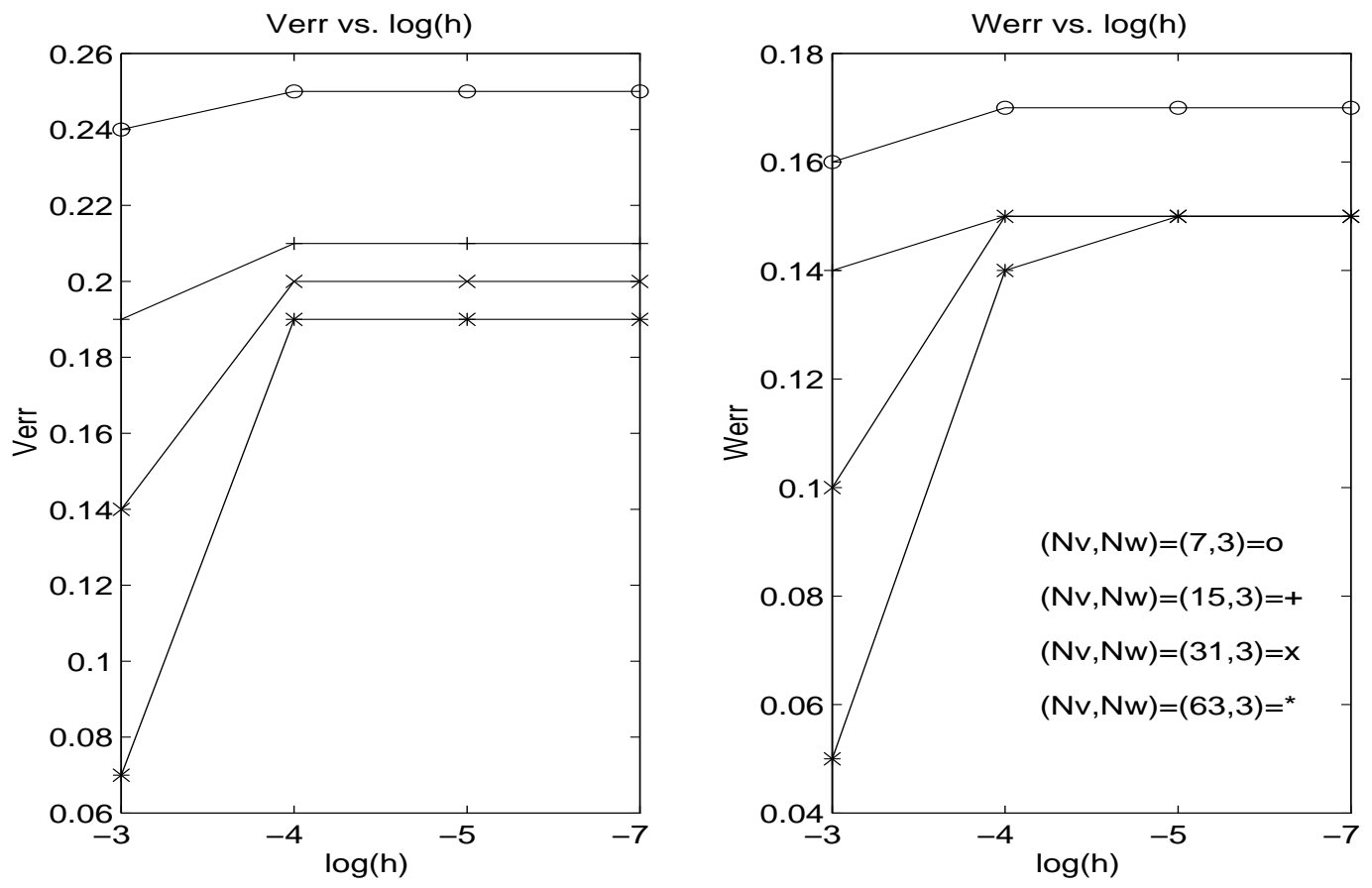

Figure 5.1 Errors vs. h (radial load) for the Linear/Cubic method 
For each mesh $N=\left(N_{v}, N_{w}\right)$, the relative errors, Verr and Werr, become nondecreasing as the thickness reduces, similar to what we have observed in Case 1. We investigate the beam with thickness $h=5.2 e-5$ further as the mesh can be refined (Table 5.3).

\begin{tabular}{||c|c||l|l|l||}
\hline Verr & Werr & $N_{v}$ & $N_{w}$ & K-cond \\
\hline .19 & .15 & 127 & 3 & $2.1 \mathrm{e}+7$ \\
\hline .19 & .15 & 255 & 3 & $3.0 \mathrm{e}+8$ \\
\hline .33 & .19 & 255 & 15 & $5.2 \mathrm{e}+8$ \\
\hline .35 & .19 & 255 & 255 & $2.7 \mathrm{e}+9$ \\
\hline .33 & .18 & 511 & 31 & $8.3 \mathrm{e}+10$ \\
\hline .30 & .17 & 1023 & 31 & $1.1 \mathrm{e}+11$ \\
\hline
\end{tabular}

Table 5.3: Simulation (radial load) with Linear/Cubic method for beam with $h=5.2 e-5$

We observe that little improvement is produced with finer meshes. In fact, the errors worsened at $N=(255,15)$. Since there is no obvious "hidden" constraint, we do not choose to classify these difficulties as some type of locking. Instead, we turn to other intrinsic properties of the model, such as the fact that (5.4) is singularly perturbed by a factor $h^{2}$ in the leading coefficient. When $h \rightarrow 0$, the system becomes inconsistent, i.e., from (5.3) we have that $\partial v+w$ is constant for all $h>0$, while (5.4) implies that under constant load, $\partial v+w$ becomes unbounded as $h \rightarrow 0$. The ill-posed nature of the system requires the mesh size and the perturbation factor (which is of order $h^{2}$ ) in (5.4) to be compatible in magnitudes; however in general this can not be attained in practical computations.

The simulations with the Cubic/Cubic method for beams with varying thickness from $h=5.2 e-3$ to $h=5.2 e-7$ were also studied (Table 5.4). We observe that the relative errors are magnitudes smaller than those from using the Linear/Cubic method (Figure 5.1) and that the errors do converge for all thickness even for badly conditioned stiffness matrices. We conjecture that methods with high order basis functions, such as cubic B-splines, can produce acceptable approximations for smaller thickness values than those needed with lower order elements.

In summary, it is readily seen that for either $\hat{q}_{\theta}$ or $\hat{q}_{n}$ constant in $h$, there are difficulties as $h \rightarrow 0$ associated with ill-posedness in the physical models (but not in the approximation schemes). Furthermore, if we follow common practice and choose loads as functions of the thickness parameters, we see that extensional loading $\left(\hat{q}_{\theta}\right)$ can lead to numerical difficulties which can be associated with a hidden constraint and therefore could justifiably be classified as a type of locking. This does not occur with normal loading $\left(\hat{q}_{n}\right)$ of the curved beam. 


\begin{tabular}{||c|c|c||l|l|c||}
\hline thickness & Verr & Werr & $N_{v}$ & $N_{w}$ & K-cond \\
\hline $5.2 \mathrm{e}-3$ & $8.0 \mathrm{e}-3$ & $3.0 \mathrm{e}-3$ & 5 & 3 & $9.39 \mathrm{e}+02$ \\
\cline { 2 - 6 } & $4.0 \mathrm{e}-3$ & $3.0 \mathrm{e}-3$ & 9 & 7 & $2.48 \mathrm{e}+04$ \\
\cline { 2 - 6 } & $2.0 \mathrm{e}-4$ & $8.0 \mathrm{e}-5$ & 17 & 15 & $1.01 \mathrm{e}+05$ \\
\hline $5.2 \mathrm{e}-5$ & $8.0 \mathrm{e}-3$ & $3.0 \mathrm{e}-3$ & 5 & 3 & $1.12 \mathrm{e}+03$ \\
\cline { 2 - 6 } & $3.0 \mathrm{e}-2$ & $2.0 \mathrm{e}-2$ & 9 & 7 & $7.44 \mathrm{e}+05$ \\
\cline { 2 - 6 } & $6.0 \mathrm{e}-2$ & $4.0 \mathrm{e}-2$ & 17 & 15 & $1.76 \mathrm{e}+08$ \\
\cline { 2 - 6 } & $3.7 \mathrm{e}-4$ & $2.1 \mathrm{e}-4$ & 65 & 63 & $5.68 \mathrm{e}+10$ \\
\hline $5.2 \mathrm{e}-7$ & $8.0 \mathrm{e}-3$ & $3.0 \mathrm{e}-3$ & 5 & 3 & $2.17 \mathrm{e}+3$ \\
\cline { 2 - 6 } & $3.0 \mathrm{e}-2$ & $2.0 \mathrm{e}-2$ & 9 & 7 & $1.56 \mathrm{e}+6$ \\
\cline { 2 - 6 } & $6.0 \mathrm{e}-2$ & $4.0 \mathrm{e}-2$ & 17 & 15 & $4.5 \mathrm{e}+8$ \\
\cline { 2 - 6 } & $7.0 \mathrm{e}-2$ & $5.0 \mathrm{e}-2$ & 33 & 31 & $1.2 \mathrm{e}+11$ \\
\cline { 2 - 6 } & $3.0 \mathrm{e}-2$ & $2.0 \mathrm{e}-2$ & 129 & 127 & $2.0 \mathrm{e}+15$ \\
\hline
\end{tabular}

Table 5.4: Simulation (radial load) with Cubic/Cubic method for beam with decreasing thickness

\section{Conclusion}

In summarizing our findings here, we make several remarks. In developing computational methods for the coupled tangential and radial motions in active curved beams, a natural choice (based on the weak formulation (3.2)) is the Linear/Cubic method described above. However, this method leads quickly to numerical difficulties and is not a particularly good choice. The Cubic/Cubic method, while requiring more effort and larger bandwidths in the approximating matrices, is generally a better choice.

A second result has even more direct implications for smart material structures and piezo actuation and sensing. Small piezo patches contribute significantly (shifts up to $15 \%$ and $20 \%$ ) to fundamental frequencies and alter in a nontrivial manner the mode shapes of the passive structure. Thus, in developing identification, control and other system theoretic methodologies for smart material structures, the passive contributions of even small piezo patches to mass, stiffness, and damping should not be ignored as this will lead to incorrect frequencies and mode shapes.

\section{Acknowledgment}

We are grateful to Dr. Geof Tomlinson for discussions which further stimulated our interest in computational methods for smart material structures with curvature. We also wish to thank Dr. Yun Wang and Dr. Ralph Smith for numerous helpful discussions. 


\section{References}

[A] D.N. Arnold, Discretization by finite elements of a model parameter dependent problem, Numerische Mathematik, 37 (1981), pp. 405-421.

[A1] R.R. Archer, Small vibrations of thin incomplete circular rings, Intern. J. Mech. Sci., 1(1996), pp. 45-56.

[ACCHW] P. Akella, X. Chen, W. Cheng, D. Hughes and J.T. Wen, Modeling and control of smart structures with bonded piezoelectric sensors and actuators, Smart Materials and Structures, 3 (1994), pp. 344-353.

[AF] D.N. Arnold and R.S. Falk, A uniformly accurate finite element method for the Reissner-Mindlin plate, SIAM J. of Numerical Analysis, 26 (1989), pp. 1276-1290.

[ATW] B. Azvine, G.R. Tomlinson and R.J. Wynne, Initial studies in the use of active constrained layer damping for controlling resonant vibration. Presented at the SPIE Conference on Smart Structures and Materials, Orlando, 1994; Smart Materials and Structures, submitted.

[BBF] F. Brezzi, K.-J. Bathe and M. Fortin, Mixed-interpolated elements for Reissner-Mindlin plates, International J. for Numerical Methods in Engineering, 28 (1989), pp. 1787-1801.

[BILW] H.T. Banks, D.J. Inman, D.J. Leo and Y. Wang, An experimentally validated damage detection theory in smart structures, CRSC-TR95-7, 1995, N.C. State University; J. Sound and Vibration, 191 (1996), pp. 859-880.

[BIW] H.T. Banks, K. Ito and Y. Wang, Well-posedness for damped second order systems with unbounded input operators, CRSC-TR93-10, 1993, N.C. State University; Differential and Integral Equations, 8 (1995), 587-606.

[BK] H.T. Banks and K. Kunisch, Estimation Techniques for Distributed Parameter Systems, Birkhaüser, Boston, 1989.

[BMZ] H.T. Banks, N.G. Medhin and Y. Zhang, A mathematical framework for curved active constrained layer structures: well-posedness and approximation, CRSC-TR95-32, 1995, N.C. State University; Numerical Functional Analysis \& Optimizations, 17 (1996), pp. 1-22.

[BS] H.T. Banks and R. Smith, Implementation issues regarding PDE-based controllers-control of transient and periodic plate vibrations, CRSC-TR95-16. 1995, N.C. State University; SIAM J. Control and Optimization, to appear. 
[BS1] I. Babuŝka and M. Suri, On locking and robustness in the finite element method, SIAM J. of Numerical Analysis, 29 (1992), pp. 1261-1293.

[BSW] H.T. Banks, R.C. Smith and Y. Wang, Smart Material Structures: Modeling, Estimation and Control, Masson/J. Wiley, Paris, 1996.

[BSW1] H.T. Banks, R.C. Smith and Y. Wang, The modeling of piezoceramic patch interactions with shells, plates, and beams, CRSC-TR92-12, 1992, N.C. State University; Quarterly of Applied Mathematics, 53 (1995) , pp. 353-381.

[CA] E.F. Crawley and E.H. Anderson, Detailed models of piezoceramic actuation of beams, AIAA Paper 89-1388-CP, 1989.

[CFW] R.L. Clark, Jr., C.R. Fuller and A. Wieks, Characterization of multiple piezoelectric actuators for structural excitation, J. Acoust. Soc. Am., 90 (1991), pp. 346-357.

[CL] E.F. Crawley and J. de Luis, Use of piezoelectric actuators as elements of intelligent structures, AIAA Journal, 25 (1987), pp. 1373-1385.

[DFR] E.K. Dimitriadis, C.R. Fuller and C.A. Rogers, Piezoelectric actuators for distributed noise and vibration excitation of thin plates, ASME Journal of Vibration and Acoustics, 13 (1991), pp. 100-107.

[DIG] J. Dosch, D.J. Inman and E. Garcia, A self-sensing piezoelectric actuator for collocated control, Journal of Intelligent Material Systems and Structures, 3 (1992), pp. 166-185.

[FGS] C.R. Fuller, G.P. Gibbs and R.J. Silcox, Simultaneous active control of flexural and extensional waves in beams, Journal of Intelligent Material Systems and Structures, 1 (1990), pp. 235-247.

[FH] M. Fripp and N. Hagood, Comparison of electrostrictive and piezoceramic actuators for vibration suppression, Proceedings of the 1995 North American Conference on Smart Structures and Materials, February 26-March 3, San Diego, CA, 1995.

[GT] M.V. Gandhi and B.S. Thompson, Smart Materials and Structures, Chapman and Hall, New York, 1992.

[HF] N.W. Hagood and A. von Flotow, Damping of structural vibrations with piezoelectric materials and passive electrical networks, Journal of Sound and Vibration, 146 (1992), pp. 243-268.

[JR] J. Jia and C.A. Rogers, Formulation of a laminated shell theory incorporating embedded distributed actuators, ASME, Reprinted from AD-Vol. 15, Adaptive Structures, Editor: B.K. Wada, Book No. H00533, 1989. 
[LP] Y. Leino and J. Pitkäranta, On the membrane locking of h-p finite elements in a cylindrical shell problem, International J. for Numerical Methods in Engineering, 37 (1994), pp. 1053-1070.

[RWTW] J.A. Rongong, J.R. Wright, G.R. Tomlinson and R.J. Wynne, Modeling of hybrid constrained layer/piezoceramic approach to active damping ,1995, preprint.

[SBS] M. Suri, I. Babus̃ska and C. Schwab, Locking effects in the finite element approximation of plate models, Mathematics of Computation, 64 (1995), pp. 461-482.

[TG] H.S. Tzou and M. Gadre, Theoretical analysis of a multi-layered thin shell coupled with piezoelectric actuators for distributed vibration controls, Journal of Sound and Vibration, 132 (1989), pp. 433-450.

[Z] Z-z. Zhang, Locking and robustness in the finite element method for circular arch problem, Numerische Mathematik, 69 (1995), pp. 509-522. 